\title{
Computational study of radial particle migration and stresslet distributions in particle-laden turbulent pipe flow ${ }^{\star}$
}

\author{
A. Gupta ${ }^{1, a}$, H.J.H. Clercx ${ }^{1}$, and F. Toschi ${ }^{1,2,3}$ \\ 1 Department of Applied Physics, Eindhoven University of Technology, Den Dolech 2, 5600 MB, Eindhoven, Netherlands \\ 2 Istituto per le Applicazioni del Calcolo, Consiglio Nazionale delle Ricerche, Via dei Taurini 19, 00185, Rome, Italy \\ 3 Department of Mathematics and Computer Science, Eindhoven University of Technology, Den Dolech 2, 5600 MB, Eindhoven, \\ Netherlands
}

Received 1 October 2017 and Received in final form 18 January 2018

Published online: 21 March 2018

(C) The Author(s) 2018. This article is published with open access at Springerlink.com

\begin{abstract}
Particle-laden turbulent flows occur in a variety of industrial applications as well as in naturally occurring flows. While the numerical simulation of such flows has seen significant advances in recent years, it still remains a challenging problem. Many studies investigated the rheology of dense suspensions in laminar flows as well as the dynamics of point-particles in turbulence. Here we employ a fully-resolved numerical simulation based on a lattice Boltzmann scheme, to investigate turbulent flow with large neutrally buoyant particles in a pipe flow at low Reynolds number and in dilute regimes. The energy input is kept fixed resulting in a Reynolds number based on the friction velocity around 250. Two different particle radii were used giving a particle-pipe diameter ratio of 0.05 and 0.075 . The number of particles is kept constant resulting in a volume fraction of $0.54 \%$ and $1.83 \%$, respectively. We investigated Eulerian and Lagrangian statistics along with the stresslet exerted by the fluid on the spherical particles. It was observed that the high particle-to-fluid slip velocity close to the wall corresponds locally to events of high energy dissipation, which are not present in the single-phase flow. The migration of particles from the inner to the outer region of the pipe, the dependence of the stresslet on the particle radial positions and a proxy for the fragmentation rate of the particles computed using the stresslet have been investigated.
\end{abstract}

\section{Introduction}

Two-phase flows dispersed with either particles or bubbles are relevant to many natural and industrial problems and under most practical conditions these flows are turbulent $[1,2]$. Some prominent examples involve turbulent sediment transport in river basins, avalanches, dust storms, aerosol and pollutant transport, combustion, slurries and several important industrial processes. In most of the cases, the dispersed phase exists as non-living objects, e.g. sand particles in dust storms, however there are also situations where the transported dispersed phase is formed by living cells, e.g. algae in bioreactors or plankton in marine ecosystems $[3,4]$. Photo-bioreactors can be used for the mass production of microalgae which can be processed further to make chemicals, nutrients and even biodiesel. In photo-bioreactors, apart from the dynamics of particles, it is also important to know the effect of hydrodynamic stresses on the cell membrane as these might have detrimental effects and even destroy cells [5]. Cell

\footnotetext{
* Contribution to the Topical Issue "Fluids and Structures Multi-scale coupling and modeling" edited by Luca Biferale, Stefano Guido, Andrea Scagliarini, Federico Toschi.

a e-mail: a.gupta-1@tue.nl
}

damage due to shear can represent one of the factors limiting the growth of algae, and thus one of the key technical issues in upscaling photo-bioreactors for micro-algae culturing $[6,7]$. In fact, turbulence in photo-bioreactors plays a dual role for algae growth, on one side enhances mixing, thus allowing a homogeneous distribution of nutrients and an appropriate exposure of algae to sunlight [8], on the other side, too intense turbulence can damage algae and reduce productivity of photo-bioreactors. Taking inspiration from the problem of microalgae damaging, the present study focuses on the fundamental physics of straining of (finite-size) particles in turbulence.

Turbulent multiphase flows are challenging fluid mechanics problems, even in the low volume fraction limit $[9$, 10]. The complexity associated to the carrier-phase turbulence is further increased by the coupling with the dispersed phase, which makes both experimental and numerical measurements of turbulent multiphase flows far more difficult than those of single-phase flows [11]. A variety of computational approaches is available for dispersed multiphase flows. Traditional computational investigations use techniques where the multiphase flow is computed only at the macroscale, and the details of the flow at the smaller scales (of the order of the particle diameter) are 
incorporated through appropriate reduced models based on Stokesian dynamics [10]. The validity of such an approach is questionable when fluctuations smaller than the particle diameter are relevant. When one needs to resolve the local flow around the particle, the fully-resolved approach is an alternative method where the interface between the particle and fluid domains is considered and the hydrodynamic force acting on each particle is determined from the momentum exchange between fluid and particle. A brief literature study and the overview of the work done on fully-resolved simulations of finite-size particles in turbulent flows has been provided in $[11,12]$. Currently, there exists a variety of different schemes based on finite difference or finite-volume solution methods for the NavierStokes equations combined with efficient schemes for the implementation of the solid particle boundary conditions. A DNS study of a dilute particle-laden turbulent flow in a vertical channel with fully-resolved finite-size rigid particles using the immersed boundary method was performed in $[13,14]$. The preferential accumulation of heavier particles in an open channel and their relation with flow structures was investigated in [15]. A fictitious domain method was used in [16] to study effects of large particles on the turbulence statistics in channel flow. Recently, there has been some significant work using the immersed boundary method to study suspensions of finite-size particles in a turbulent channel flow for volume fractions varying up to $20 \%$, where researchers looked into the modification of the Eulerian flow field by the dispersed phase [17-19], as well as the modified scaling laws in the presence of the particles [20]. The effect of particles on the transition to turbulence in wall-bounded flows has also been studied by several researchers [21-23]. The lattice Boltzmann method (LBM) has been an effective alternative tool for the simulation of particle suspensions with applications in many industrial and biological problems [24-26]. The simulation of finite-size fibers in the turbulent channel flow [27] or spherical particles in isotropic turbulence [28] are some examples showcasing the versatility of the method. LBM was also used to study turbulence modification by finite-size particles in a channel flow in $[29,30]$. None of these studies using fully-resolved approaches explored the straining and fragmentation of the particles due to the stresses from the surrounding fluid. In [31-34], researchers have looked into the fragmentation of solid aggregates suspended in a turbulent flow where the aggregates are assumed to be small with respect to the Kolmogorov length scale and it is further assumed that fragmentation is caused by hydrodynamic stresses which are assumed proportional to the energy dissipation. This choice was motivated by the observation that the occurrence, in the surroundings of a small particle, of instantaneous stresses capable of inducing fragmentation is controlled by the statistical (spatial and temporal) distribution of these stresses, which is in turn dictated by the turbulent flow field. In a recent work [12], we proposed the stresslet (the symmetric component of the first moment of the force per unit area at the particle surface) as a measure to quantify the effect of a straining flow field on finite-size particles.
The fragmentation of aggregates in a particle-laden flow can be caused by two mechanisms. The first mechanism is called impact fragmentation and is caused by the collisions of the particles with other particles or walls. However, we do not consider this mechanism in the present study as we are simulating a dilute suspension of particles where the collision rate is relatively small. The second type of fragmentation is caused by particle deformation induced by the hydrodynamic stresses. Deformation of a particle generates internal stresses which can break the particle by the following two processes:

1) If the response time of the particle to deformation is very large, then fragmentation is instantaneous and termed as brittle fragmentation and the particle can be referred to as brittle.

2) If fragmentation depends on the stress history and a significant amount of energy is required to overcome deformation, then the particle can be referred to as ductile and the fragmentation is called ductile fragmentation.

Similar to the fragmentation of aggregates of particles in turbulence, microalgae and planktons can also be damaged by intense hydrodynamic shear that occurs in turbulent flows. Shear sensitivity can pose a significant problem in photo-bioreactors as the intensity of turbulence needed to generate optimal light-dark cycling is difficult to achieve without damaging algal cells $[4,6,7]$. Methods are being developed to understand and quantify the damage associated with turbulence. The effect of shear stress on the viability (ability to survive) of Chaetoceros muelleri was studied by [5] using a combination of a rheometer and dedicated shearing devices. They imposed different levels of shear stress by varying the shear rates and the medium viscosity. It was possible to quantify the effect of shear stress over a wide range, whilst preserving laminar flow conditions through the use of a thickening agent. They observed that the productivity of algae suddenly drops beyond a critical value of shear rate. Therefore, it is important to understand the interactions between algae and turbulence, in particular addressing the question of how migration of cells and the damage to them is affected by the turbulent fluctuations of flow around them. In order to be consistent with the existing literature, we will use the term fragmentation in the subsequent discussion even when we refer to the damage caused to the cells which reduces its viability. In either type of fragmentation, either for particle aggregates or living cells, the phenomenology of turbulent fragmentation is still not fully understood because of the inherent complexity of the flow field along with the intricacy of the particle morphology in determining how the hydrodynamic forces redistribute over the structure of the particle and how stresses accumulate in critical locations ultimately resulting in damage or even fragmentation of particles. Consequently, simpler models based on mean properties like energy dissipation are used to compute the fragmentation rate. We used a stresslet exerted by the fluid on rigid finite-sized particles as a proxy to study the damage caused by the surrounding flow to the microalgae and planktons. From a physical point of 
view, the stresslet is the result of the resistance of the particle to a straining motion which makes it the natural quantity to compute the fragmentation rate. We focus specifically on the brittle fragmentation regime in which the cell or aggregate is fragmented as soon as the norm of the stresslet crosses a critical value, i.e. $S T>S T_{c r}$. Besides looking into the stresslet distribution as a proxy for cell fragmentation statistics, we also investigated Eulerian and Lagrangian statistics in this article using LBM.

As first observed by Segré and Silberberg [35, 36] for a pipe flow, a neutrally buoyant particle migrates radially to a preferred position. For a pipe, particles which are initially at larger radial positions are driven inward, whereas particles closer to the centerline of the tube are driven outward. This migration has been termed tubular pinch because it causes a focusing or pinching of the concentration of particles. The migration takes place because the neutrally buoyant particles are subjected to a lift force due to their finite size and the presence of a parabolic velocity profile. The particles migrate towards an equilibrium position set by the balance between this lift force and the hydrodynamic repulsion from the wall [37]. The equilibrium position associated with the Segré and Silberberg phenomenon is at $R_{e q} / R=0.6$ in the limit of a very small pipe Reynolds number. When Re increases, particles move progressively closer to the wall ( $R_{e q}$ increases) as observed in the experiments of Segré and Silberberg [35,36] and more recently in $[38,39]$. The results confirm that the tubular pinch effect, in which particles accumulate on a narrow annulus, moves toward the wall as Re increases. Many experimental and theoretical studies addressed this migration phenomenon and they showed the existence of equilibrium positions that depend on the channel geometry, particle radius, and channel Reynolds numbers. Interestingly, another important feature observed in viscous flows is the shear-induced migration which becomes important as the volume fraction increases. When considering a pressure-driven Poiseuille flow, either in a pipe or in a channel, the particles migrate toward the centerline, i.e. from high to low shear rate regions. To avoid confusion, note that inertial migration is unrelated to the rheological phenomenon of shear-induced migration, which is driven by particle-particle interactions resulting in a non-Newtonian rheology. Inertial migration results instead from the fluid-particle interaction within the confining geometry. However, all these studies focused on the migration of particles in laminar conditions. The situation becomes more complex in turbulent flows where particle motion is constantly affected by the turbulent eddies instead of staying at an equilibrium position. In this study, we will also introduce a novel definition of particle circulation frequency using the time signal of the radial position of the particles.

\section{Numerical model and simulations}

\subsection{Flow solver}

The Lattice-Boltzmann method (LBM) has been widely used for the simulation of the fluid flow [40-42]. Though, instead of solving the Navier-Stokes equations directly, the discrete Boltzmann equation is solved to simulate the flow of a fluid given a collision model, e.g., the BhatnagarGross-Krook (BGK) model. In this technique, the fluid is replaced by fictious particle distribution functions which stream along given directions (lattice links) and collide at the lattice sites. The distribution function, $f_{i}$ is the probability of finding particles within a certain range of velocities at a certain range of locations at a given time. The collision and streaming processes are local operations and therefore it can be programmed conveniently for parallel computing. Another advantage of the LBM is the handling of complex phenomena such as moving boundaries, multiphase flows etc. without a need for a separate Lagrangian mesh. The distribution function replaces the tagging of each particle, as in molecular dynamic simulations, thereby saving the computer resources drastically. By simulating streaming and collision processes across a limited number of particles, the intrinsic particle interactions evolve the macroscopic behavior of the fluid.

The lattice Boltzmann equation reads as follows [40]:

$$
f_{i}\left(\boldsymbol{x}+\boldsymbol{e}_{i} \delta_{t}, t+\delta_{t}\right)=f_{i}(\boldsymbol{x}, t)+\frac{1}{\tau}\left(f_{i}^{e q}-f_{i}\right),
$$

where $f_{i}$ is the probability distribution function and the subscript $i$ labels a set of discrete speeds connecting the nodes of a regular lattice, $f_{i}^{e q}$ is the corresponding equilibrium distribution function, $\delta_{t}$ is the time increment and $\tau$ is the fluid relaxation time related to the kinematic viscosity as $\nu=\left(\tau-\delta_{t} / 2\right) c_{s}^{2}$, where $c_{s}$ is the speed of sound.

The description of the lattice Boltzmann method as a flow solver has been discussed in numerous texts in the past and the reader can refer to [40] or [43] for more details on the method. We used the D3Q27 BhatnagarGross-Krook (BGK) lattice Boltzmann method to simulate the fluid flow. It was observed that the D3Q27 lattice model could achieve the necessary level of rotational invariance, in terms of long-time-averaged turbulence statistics, and generate results comparable to DNS data, while the D3Q19 lattice model exhibits strong angular dependency and broken rotational invariance [12]. Similar observations on the effect of lattice models (D3Q19 versus D3Q27) on the rotational invariance of a turbulent pipe flow were made in [44].

\subsection{Modelling particle-fluid interaction}

In this section, we focus on the modeling of the finite-size particles and on their coupling with the fluid. To simulate the hydrodynamic interactions between the solid particles in the suspension and the surrounding fluid, the lattice Boltzmann model must be modified to incorporate boundary conditions imposed on the fluid by the solid particles.

The solid particles are defined by a boundary surface, which can be of any size or shape. When placed on the lattice, the boundary surface cuts some of the links between lattice nodes. The fluid particles moving along these links interact with the solid surface at boundary nodes placed 


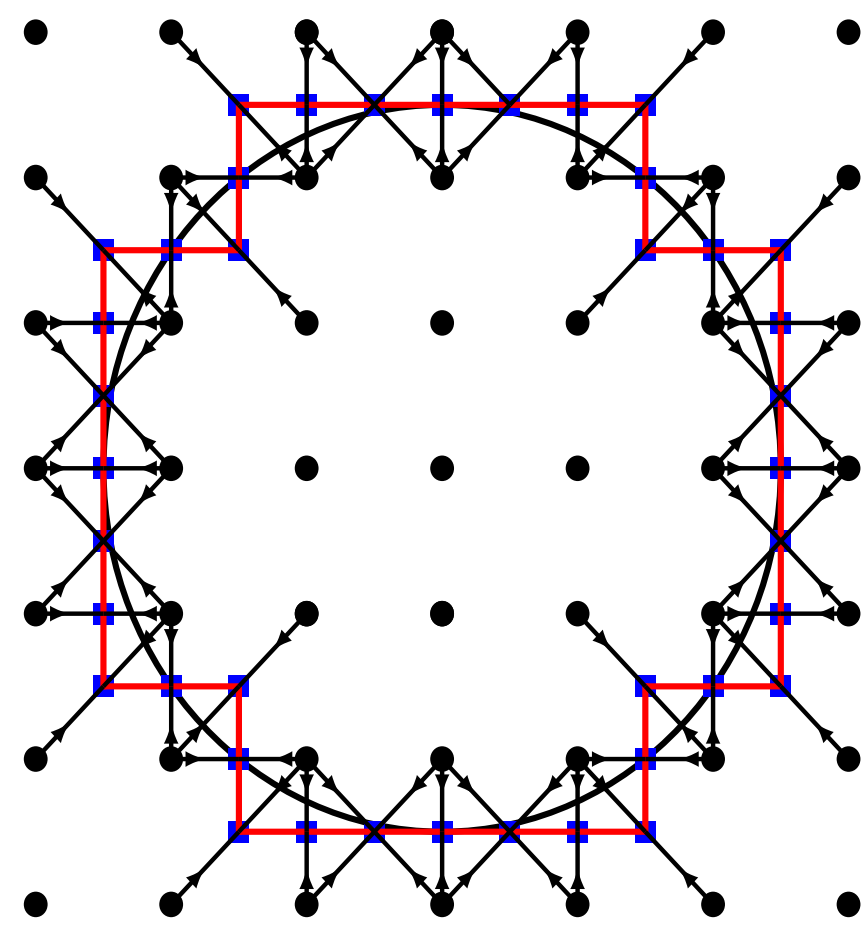

Fig. 1. Location of the boundary nodes for a circular object of radius 2.5 lattice spacings. The velocities along links cutting the boundary surface are indicated by the black arrows. The locations of the boundary nodes are shown by the blue squares and the lattice nodes by the black circles (Reproduced from [12]).

halfway along the links as shown in fig. 1. Thus, the particle boundary is approximated by a staircase shape which becomes more and more accurate as the particle resolution increases.

The solid bounce-back momentum exchange method, first proposed by Ladd [45] has been widely used in lattice Boltzmann simulations for particle-fluid interactions with several variations and improvements. In this method, nodes on either side of the boundary surface are treated in an identical fashion, so that the fluid fills the whole volume of space, both inside and outside the solid particles. The midway bounce-back means that fluid particles traveling from a fluid node towards a solid node will be bounced back on the mid-point of the boundary link

$$
f_{\bar{\alpha}}\left(\boldsymbol{x}_{f}, t+\delta_{t}\right)=\tilde{f}_{\alpha}\left(\boldsymbol{x}_{f}, t+\delta_{t}\right)-2 w_{\alpha} \rho \frac{\boldsymbol{e}_{\boldsymbol{\alpha}} \cdot \boldsymbol{u}_{\boldsymbol{w}}}{c_{s}^{2}},
$$

where $\boldsymbol{x}_{f}$ is the location of the fluid node outside the solid surface and $\boldsymbol{e}_{\boldsymbol{\alpha}}$ is the lattice direction from a fluid node to a solid node, $\boldsymbol{e}_{\overline{\boldsymbol{\alpha}}}$ represents the opposite direction, $\tilde{f}$ is the post collision distribution function and $\boldsymbol{u}_{\boldsymbol{w}}$ is the velocity of the moving boundary evaluated at the mid-point of the link.

The force acting on the moving boundary can be obtained by momentum exchange along each link

$$
\delta \boldsymbol{F}_{\boldsymbol{w}}\left(\boldsymbol{x}_{\boldsymbol{w}}, \boldsymbol{e}_{\boldsymbol{\alpha}}\right)=-\left[f_{\bar{\alpha}}\left(\boldsymbol{x}_{f}, t\right) \boldsymbol{e}_{\overline{\boldsymbol{\alpha}}}-\tilde{f}_{\alpha}\left(\boldsymbol{x}_{f}, t+\delta_{t}\right) \boldsymbol{e}_{\boldsymbol{\alpha}}\right] .
$$

The fluid within the particle boundary was kept for computational convenience only, since it avoids the necessity of creating and destroying fluid as the particle moves. In this formulation, where particles are treated as shells containing fluid, the fluid inside the particles has the potential to affect particle dynamics. In order to run simulations with neutrally buoyant particles, the mass of the shell containing the interior fluid must approach zero. This creates instabilities in the particle update procedure. Consequently, this method was limited to solid particles with density larger than the fluid density. This problem was overcome by proposing a model in which there is no fluid to occupy the solid particle volume, fluid nodes appear and disappear as the solid particle moves through the domain [46]. The covering and uncovering of nodes in this scheme results in a significantly fluctuating impulse force on the particles, which may reduce the simulation stability. Chen et al. [47] observed that for particle suspension models without interior fluid, if the impulse force is not applied, the simulation results deviate from the FEM (Finite-Element Method) result. They discussed the physical cause of this problem, and found that the initial momentum of the net mass transfer through boundaries in the moving-boundary treatment is not counted in the conventional momentum exchange method. A corrected momentum exchange method is then proposed by taking into account the initial momentum of the net mass transfer at each time step. The actual momentum exchange along each boundary link is obtained by calculating the momentum difference of the outgoing fluid particles and ingoing fluid particles. The initial macroscopic velocity of the net mass transfer, $-2 w_{\alpha} \rho \frac{\boldsymbol{e}_{\alpha} \cdot \boldsymbol{u}_{\boldsymbol{w}}}{c_{s}^{2}}$, is approximately the velocity of the solid wall but not zero as long as the wall is moving. The conventional momentum exchange method does not count in the initial momentum of the net mass transfer, $\left(-2 w_{\alpha} \rho \frac{\boldsymbol{e}_{\boldsymbol{\alpha}} \cdot \boldsymbol{u}_{\boldsymbol{w}}}{c_{s}^{2}}\right) \boldsymbol{u}_{\boldsymbol{w}}$. As a result, the impulse force has to be applied to the conventional momentum exchange method as a correction. This could also be the reason why non-shell models (models without fluid inside particle) with the conventional momentum exchange method breaks Galilean invariance. Then it is possible to correct the conventional momentum exchange method by simply accounting for the initial momentum of the net mass transfer

$\delta \boldsymbol{F}_{\boldsymbol{w}}\left(\boldsymbol{x}_{\boldsymbol{w}}, \boldsymbol{e}_{\boldsymbol{\alpha}}\right)=-\left\{f_{\bar{\alpha}}\left(\boldsymbol{x}_{f}, t\right) \boldsymbol{e}_{\overline{\boldsymbol{\alpha}}}-\left[\tilde{f}_{\alpha}\left(\boldsymbol{x}_{f}, t+\delta_{t}\right) \boldsymbol{e}_{\boldsymbol{\alpha}}+\Delta M\right]\right\}$,

where $\Delta M$ is the initial momentum of the net mass transfer

$$
\Delta M=\left(-2 w_{\alpha} \rho \frac{\boldsymbol{e}_{\boldsymbol{\alpha}} \cdot \boldsymbol{u}_{\boldsymbol{w}}}{c_{s}^{2}}\right) \boldsymbol{u}_{\boldsymbol{w}}
$$

Mid-point bounce-back proposed by Chen et al. [47] for the analysis of solid particles with inertia suspended in a fluid was used for particle-fluid coupling in this work. With the net force and the torque calculated using the momentum exchange, the motion of the solid particle is simply determined by solving Newton's equations for the linear and angular momentum. In the simulations discussed here, these equations are solved using a leap-frog integration scheme to obtain the complete motion of the suspended solid particles in the fluid. The angular dynamics was inte- 
grated by the method of quaternions, as discussed in [48], for representing orientations and rotations of finite-size particles.

The numerical method is capable of modeling the interaction among the particles also when their gap distance is below the grid size using a lubrication force based on the theoretical lubrication approximation for a small gap between regular shaped objects. A complete discussion of these models can be found in [12], where several test cases are presented as validation. We provide here only evidence for the ability of the present numerical tool to accurately compute the stresslet exerted by fluid in a laminar pipe flow. In the simulation, a spherical particle of radius $r_{p}$ is placed at different radial positions in a pipe of diameter $D$. The length of the pipe is twice its diameter and the flow is driven by a constant body force mimicking the pressure difference. The Reynolds number based on the friction velocity and the diameter of the pipe $\left(R e_{\tau}=u_{\tau} D / \nu\right)$ is 0.88 . Periodic boundary condition is used in axial direction while the no-slip boundary condition is used along the lateral directions with the system resolution being $240 \times 120 \times 120$. The results are compared with the values computed using Faxén laws which provide an analytical expression for stresslet under low Reynolds number conditions in the dilute regime and away from the boundaries:

$$
\mathbf{S}=\frac{20}{3} \pi \mu r_{p}^{3}\left(1+\frac{r^{2}}{10} \nabla^{2}\right) \mathbf{E}(x=0)
$$

In the above expression, $r_{p}$ is the radius of the particle and $\mathbf{E}$ is the rate of strain in ambient flow. The $x=0 \mathrm{im}-$ plies that the ambient fields are evaluated at the position occupied by the center of particle when the particle is not present. The simulations run for 20000 time steps (in lattice units) until a steady state is reached. We computed the ratio of the norm of the stresslet $S T_{N}$ to the prefactor computed from Faxén's expression of the stresslet $\left(S T_{o}=\frac{20}{3} \pi \mu r^{3}\right)$ from the simulation (in steady state) and compare our results with the analytical results. The radial position of the particle is moved gradually closer to the wall to understand the wall effect on the radial stresslet profile. The results obtained from the simulation converges to the analytical values for the particle radial position close to the center of the pipe as shown in fig. 2 while the deviations are observed near the wall. The deviation from the analytical results are due to the close proximity of the particle surface with the pipe wall where Faxén's law does not hold anymore. Nevertheless, it was observed that the error remains below $5 \%$ for a radial position of the sphere (denoted by $r / R$ ) less than 0.7 .

The validation discussed above holds for a Reynolds number approaching zero because we were making a comparison with analytical results obtained for low Reynolds number. Since we discuss simulations for higher Reynolds number, we present here another validation for a spherical particle placed symmetrically in a Couette flow at finite Reynolds number. We consider a neutrally buoyant rigid particle immersed in a fluid. We denote the flow direction by 1 , the velocity gradient direction by 2 , and the vorticity direction by 3 . In figs. 3 and 4 , we present various rheolog-

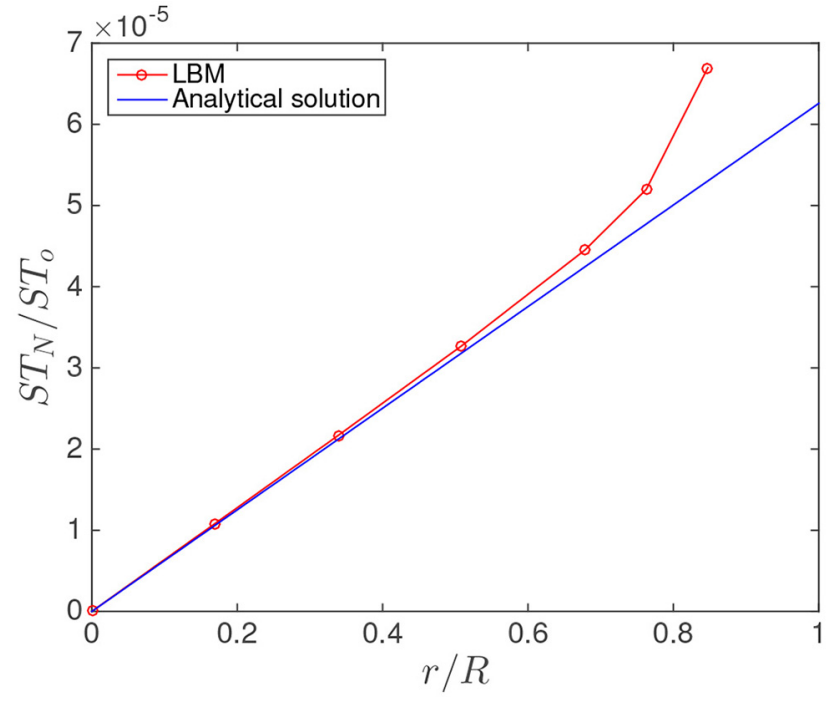

Fig. 2. Norm of stresslet tensor on a sphere in a Poiseuille flow, along with the solution obtained from Faxén laws. The result deviates from the analytical solution close to the wall due to the close proximity of the particle surface with the pipe wall where Faxén's law does not hold anymore.

ically interesting quantities resulting from the stresslet on a sphere. These include the shear component, $S_{12}$, the first $\left(S_{11}-S_{22}\right)$ and second $\left(S_{22}-S_{33}\right)$ normal stress difference contributions, and the trace of the stresslet given by $\left(S_{11}+S_{22}+S_{33}\right) / 3$. The last is a quantity related to what is termed the suspension pressure. The results are normalized using $\nu G r_{p}^{3}$, where $\nu$ is the viscosity of the medium, $G$ is the shear rate and $r_{p}$ is the radius of the particle. We compared the theoretical predictions of both $[49,50]$ with our numerical results. The predictions from [49] are

$$
\begin{aligned}
S_{12} & =\frac{10 \pi}{3}+5.61 R e^{3 / 2}, \\
S_{11}-S_{22} & =-\frac{16 \pi}{9} R e+1.202 R e^{3 / 2}, \\
S_{22}-S_{33} & =-\frac{409 \pi}{315} R e-1.056 R e^{3 / 2} ;
\end{aligned}
$$

and those of [50] are

$$
\begin{aligned}
S_{12} & =\frac{10 \pi}{3}+1.82 R e^{3 / 2}, \\
S_{11}-S_{22} & =-\frac{16 \pi}{9} R e+3.343 R e^{3 / 2}, \\
S_{22}-S_{33} & =-\frac{409 \pi}{315} R e-1.558 R e^{3 / 2}, \\
\frac{1}{3} S_{i i} & =\frac{19 \pi}{36} R e .
\end{aligned}
$$

Our results matches well the theoretical results except for the shear component at higher Reynolds number. However, the deviations in $S_{12}$ are similar to those observed by [51] using a finite-element method. The error for the numerical simulations might be attributed to the finite system size and particle resolution. 

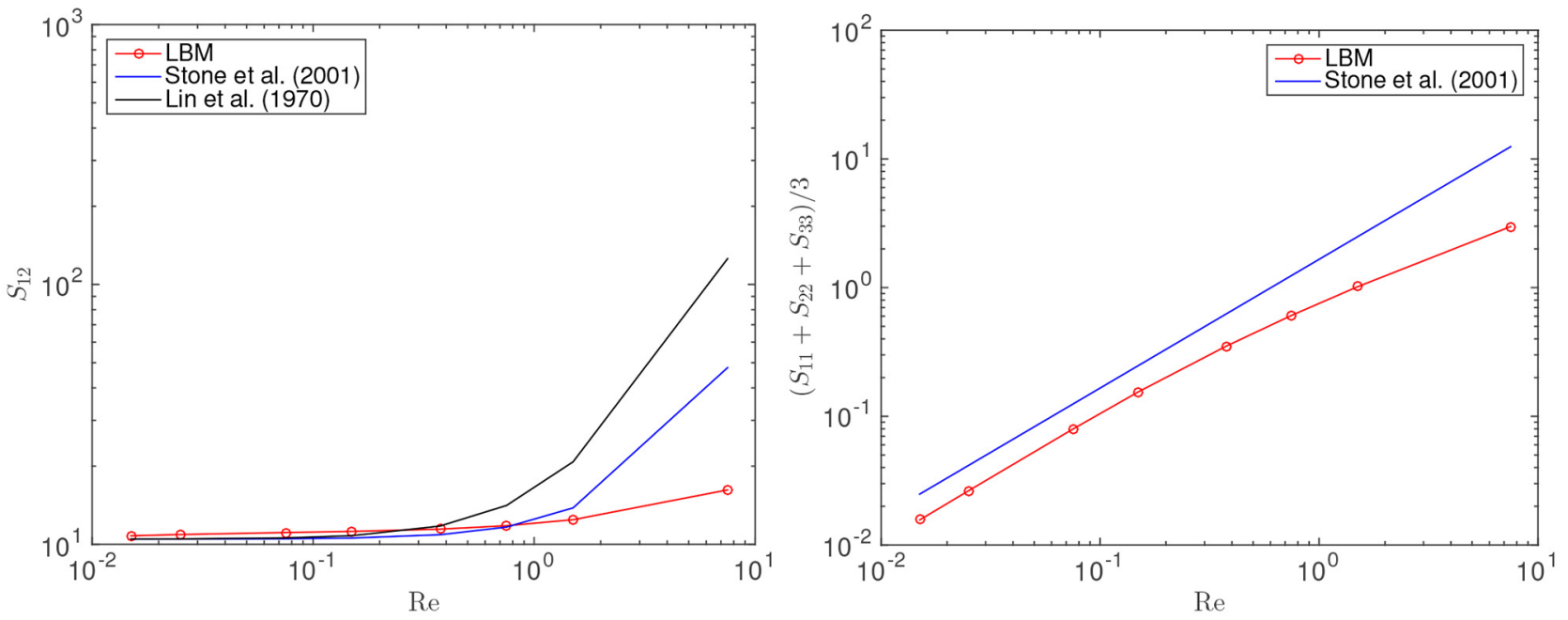

Fig. 3. The shear component, $S_{12}$ (left) and the trace of the stresslet, $\left(S_{11}+S_{22}+S_{33}\right) / 3$ (right), scaled by $\nu G r_{p}^{3}$, where $\nu$ is the viscosity of the medium, $G$ is the shear rate and $r_{p}$ is the radius of the particle.
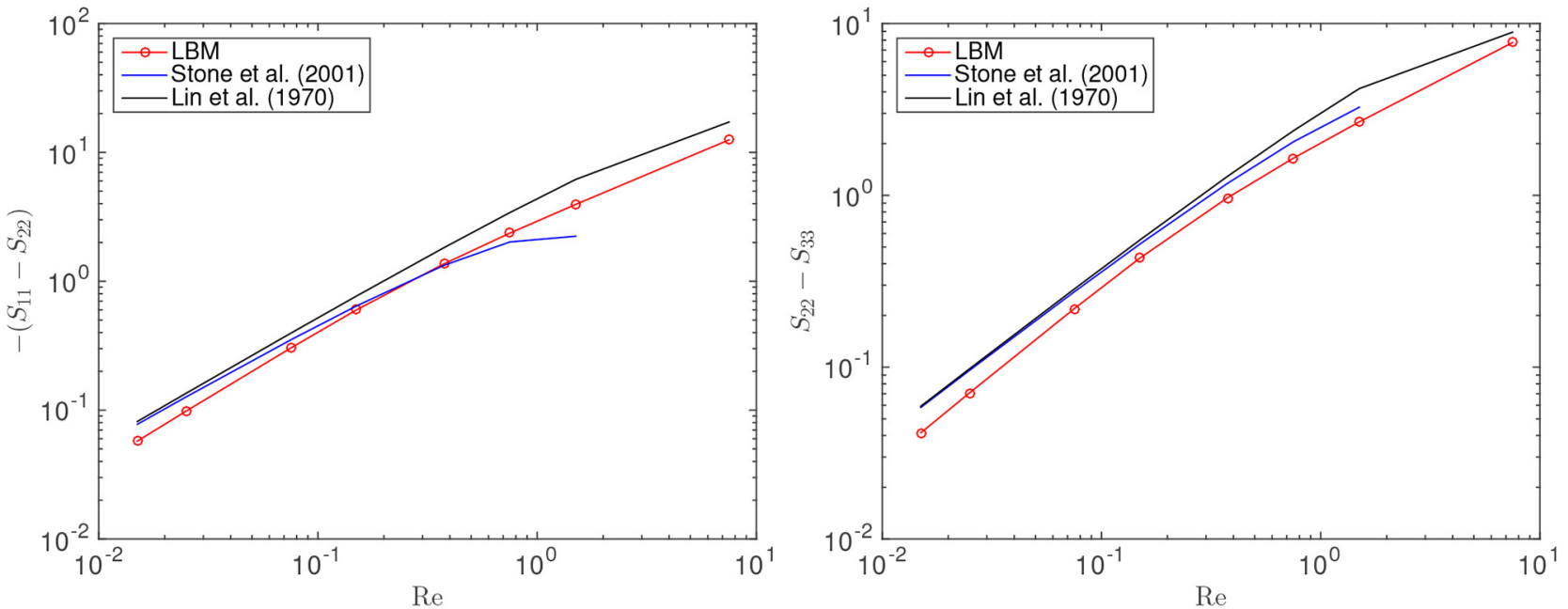

Fig. 4. The first, $\left(S_{11}-S_{22}\right)$ (left) and second, $\left(S_{22}-S_{33}\right)$ (right) normal stress difference contributions, scaled by $\nu G r_{p}^{3}$, where $\nu$ is the viscosity of the medium, $G$ is the shear rate and $r_{p}$ is the radius of the particle.

\subsection{Simulations of turbulent pipe flow}

The turbulent pipe flow is simulated via a circular duct element with length $L=4 R$ where $R$ is the pipe radius and periodic inlet/outlet boundary conditions. We used the mid-point bounce-back boundary condition for the solid pipe boundary to impose the no-slip condition on the cylindrical wall [43]. The domain is discretized by a cubic mesh of $480 \times 240 \times 240$ with 480 lattice points in the stream-wise and 240 in the lateral directions. In fig. 8 , sect. 3, we have shown a visualization of the computational domain along with the coordinate system.

The Reynolds number based on the friction velocity and the diameter of the pipe $\left(R e_{\tau}=u_{\tau} D / \nu\right)$ are used in the subsequent discussion. The flow is characterized by a nearly constant energy input controlled by a varying body force, $g$, in the axial direction. In order to make a quantitative comparison between the flow with and with- out particles, we decided to keep the energy input in the flow as constant. This is achieved by varying the volume force $\boldsymbol{g}=(g, 0,0)$ at each time step in order to obtain a fixed energy input $E_{i n}=\langle\boldsymbol{g} \cdot \boldsymbol{U}\rangle_{1-\phi}=10^{-8}$, where $\boldsymbol{U}=\left(U_{x}, U_{r}, U_{\theta}\right)$ is the velocity of the fluid, $\phi$ is the volume fraction of the particles and $\langle\cdot\rangle_{1-\phi}$ represents the fluid averaged dot product, i.e., the nodes within the particle boundaries are excluded from the average. Two cases with particle radii of 6 and 9 lattice units $(l u)$ were considered, giving a particle-pipe diameter ratio of 0.05 and 0.075 respectively. The case with particle radius $r_{p}=6 l u$ will be referred to as $\mathrm{R} 6$, and the case with particle radius $r_{p}=9 l u$ will be referred to as R9 in the subsequent discussion. The number of particles was kept same in both cases $\left(N_{p}=128\right)$ giving a volume fraction of $0.54 \%$ and $1.83 \%$ for the R6 and R9, respectively. These two simulations are complemented with a reference single-phase flow (R0): the unladen case with the same energy input. The simulation 
Table 1. Parameter settings for the turbulent simulations.

\begin{tabular}{llllll}
\hline $\mathrm{R}$ & Volume fraction & Forcing & $R e_{\tau}$ & $u_{\tau}$ & $t^{+}$ \\
\hline 0 & 0 & $2.06 \times 10^{-7}$ & 250 & 0.0035 & 33990 \\
6 & $0.54 \%$ & $2.16 \times 10^{-7}$ & 256 & 0.00358 & 33190 \\
9 & $1.83 \%$ & $2.23 \times 10^{-7}$ & 260 & 0.00364 & 32670 \\
\hline
\end{tabular}

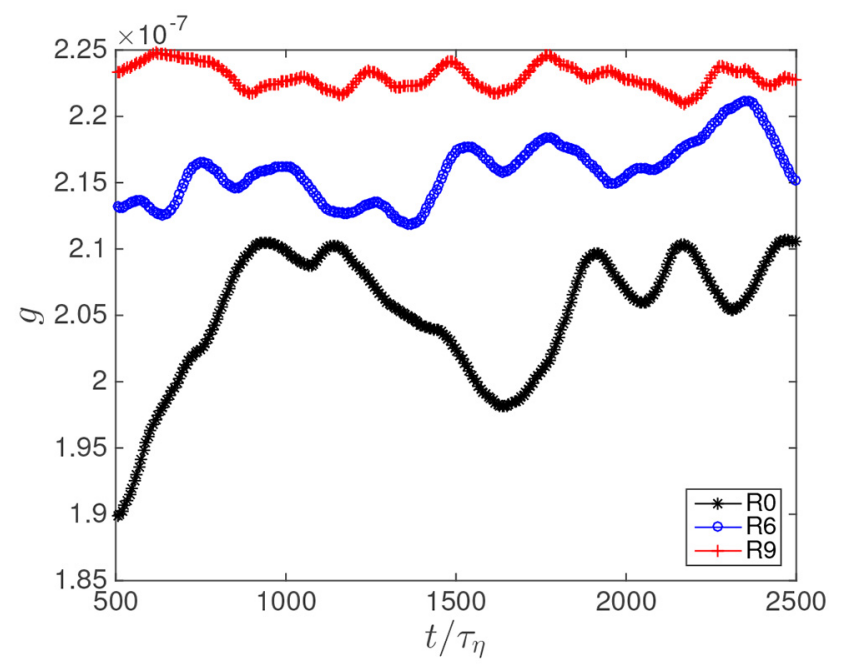

Fig. 5. Time history of the forcing signal for the three cases needed to obtain fixed energy input $E_{i n}=\langle\boldsymbol{g} \cdot \boldsymbol{U}\rangle_{1-\phi}=10^{-8}$.

starts from a laminar flow and with a random positioning of the particles. Transition to turbulence occurs at fixed Reynolds number because of the fluctuations added by the presence of the particles. Statistics are collected after the initial transient phase when the flow reaches a pseudosteady state. The integral time scale is computed using the pipe radius and friction velocity $t^{+}=R / u_{\tau}$ and is shown in table 1.

Figure 5 shows the variation of the forcing as a function of time (excluding the initial transient phase) for the three cases considered. As expected, larger forcing is needed for R9, probably due to larger viscous dissipation caused by the particle surface. However, in addition to the increased surface area, there are lot of factors at play which might result in the higher forcing required for $\mathrm{R} 9$, including the higher volume fraction along with the complex interaction between the particles and the surrounding fluid. Table 1 also shows the mean forcing values which are used to compute the respective Reynolds number.

The Kolmogorov scales were computed using the energy input assuming equilibrium, i.e., $E_{i n}=\epsilon$ (mean energy dissipation). Since energy input and viscosity are kept constant in all the simulations, we get a Kolmogorov length scale $\eta=1.38$ and a Kolmogorov time scale $\tau_{\eta}=$ 577 in lattice units for all the simulations. This value of the Kolmogorov length scale satisfies the constraint $\pi \eta \geq 1$ and $\tau_{\eta}>1$, which is one of the criteria for good quality of the DNS. Another criterion to ensure fully-resolved simulations concerns the size of the computational domain which should be sufficiently large to accommodate

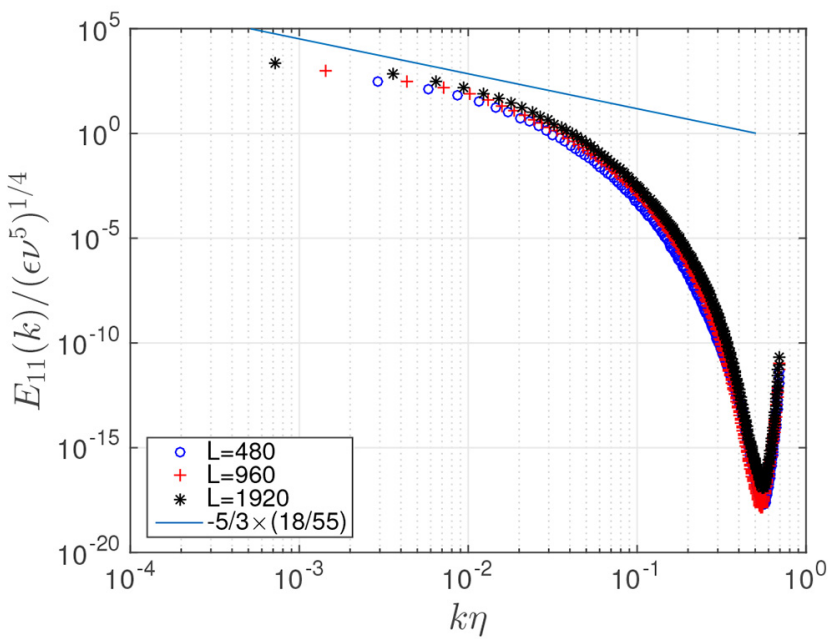

Fig. 6. One-dimensional energy spectrum for turbulent fluctuations along the axial direction in the bulk of the turbulent pipe without particles normalized by the energy dissipation and kinematic viscosity against the wavenumber normalized by the Kolmogorov length scale for different length of the pipe.

all relevant large-scale structures. This was studied in [52] and they found that the radial and circumferential twopoint correlation coefficients of the three fluctuating velocity components decay to zero within a pipe length equal to the pipe diameter. Since the flow is periodic in the axial direction, this suggests that $L=4 R$ as used in our simulations is sufficient to resolve all flow structures in the lateral direction. However, they also observed that the axial twopoint correlation coefficient for velocity fluctuations does not decay to zero even after $L=5 R$ (corresponding to a pipe length of $L=10 R$ ) in the near wall region. This is due to the streamwise elongated flow structures present in the near-wall region which causes the correlations to be non-zero. In [18] and [22], it is suggested that the presence of particles tends to disrupt long flow structures so that the effect on particle dynamics would not be different in longer domains (the influence of particles on flow structures is further discussed in sect. 9). In [53], a fairly good agreement with the results in [52] for $L=4 R$ was observed. Figure 6 shows the one-dimensional energy spectrum for turbulent fluctuations along the axial direction in the bulk $(0 \leq r / R \leq 0.25)$ of the turbulent pipe without particles for three different pipe lengths of $L=4 R, 8 R$ and $16 R$. The spectrum extends in the low wavenumber regime for longer pipes without any significant influence in the large wavenumber regime. Therefore, we used the pipe length equal to four times the radius $(L=4 R)$ in the present analysis.

Figure 7 shows the time history (excluding the initial transient phase) of the energy dissipation $(\epsilon)$ and of the total kinetic energy ( $T K E$ ) per unit length where a timelag between the two plots is suggestive of the presence of energy cascade. The kinetic energy has been scaled down in the plot such that its root-mean-square (RMS) value is the same as the RMS of energy dissipation. The energy dissipation has been computed as discussed in sect. 3.2. 

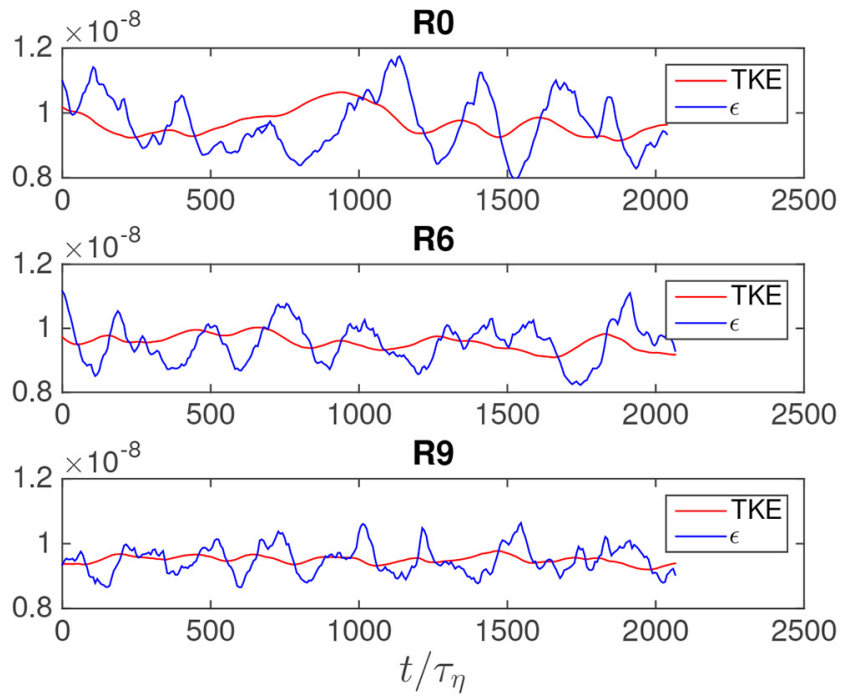

Fig. 7. Time series of the total kinetic energy $(T K E)$ and energy dissipation $(\epsilon)$ per unit length in turbulent pipe for single-phase flow (R0) and the two particle radii (R6 and R9). The kinetic energy has been scaled down in the plot such that its RMS value is the same as the RMS of energy dissipation.

\section{Turbulence characteristics of the carrier phase}

In this section, we discuss how the presence of particles affects the turbulence structure of the fluid phase with particular focus on the RMS of velocity fluctuations and on the energy dissipation. A visual impression of the suspension flow in the whole domain is provided in fig. 8, which shows a typical particle configuration along with a colour plot of the instantaneous streamwise velocity.

\subsection{RMS velocity profiles}

Here we discuss the effect of particles on the Eulerian RMS velocity profiles. In the presentation of the results, the distance from the wall, $y^{+}$, is defined as $y^{+}=\frac{D^{+}}{2}-r^{+}$, where $r$ is the radial coordinate in the cylindrical coordinate system with the pipe center as the origin, $D$ is the diameter of the pipe and the + sign denotes the normalization with wall units, $\nu / u_{\tau}$. The root-mean-square (RMS) velocity is normalized by the friction velocity, $u_{\tau}$. Figure 9 shows the axial and radial RMS velocities values for the three simulations.

Our results indicate that the presence of large particles results in a decrease of the root-mean-square (RMS) of the streamwise velocity fluctuation and in an enhancement of the RMS of radial and circumferential (not shown here) velocity fluctuations near to the wall. This indicates that the presence of particles redistributes energy more isotropically. In [53], similar results were observed and explained this by using the vortical structures in the flow. For the particle-free case, the flow field is dominated by large-scale quasi-streamwise vortices. The particles induce small-scale vortices in the near-wall region, resulting in the

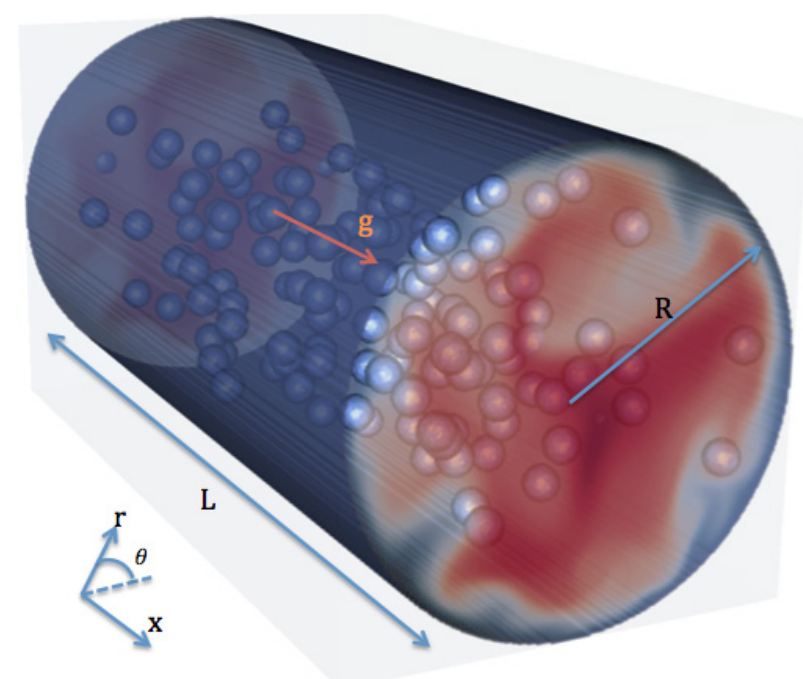

Fig. 8. Instantaneous snapshots of the streamwise velocity together with the particle position for the case R9.

enhancement of the RMS of radial and azimuthal velocity fluctuations there. At the same time, the strength of large-scale streamwise vortices is attenuated by the particles in the particle-laden cases. Therefore, the large-scale vortices exhibit more three-dimensional features due to the particle effects, unlike the particle-free case where they are mainly extended in the streamwise direction. Accordingly, the large-scale streamwise fluctuating velocities are attenuated, and the peak RMS of the streamwise velocity fluctuation is reduced in the presence of the particles while the RMS of radial velocity increases near to the wall.

\subsection{Mean profiles and energy dissipation}

We investigate in more detail the mean velocity profiles and the flow dynamics near the wall. Figure 10 depicts the mean particle and fluid velocity profiles in viscous wall units. The fluid velocity statistics have been calculated considering only the points located out of the volume occupied by the particles (phase-ensemble average). Similarly, the particle velocity profile is computed using the rigid-body motion for lattice nodes inside the particle. This means we do not just use the velocity of the center of mass of the particle but the velocity of each node within the particle to compute these statistics. As shown in fig. 10, both fluid and particles have the same mean velocity in the whole pipe with the exception of near to the wall where particles have a mean velocity larger than the surrounding fluid. While the velocity at the wall is zero for the fluid, this is not the case for the solid phase as particles can have a relative tangential motion. This relative slip and the strong effect of particles on the fluid wall layer causing a considerable modification of the streamwise velocity structures compared to singlephase flow were already observed in [18]. Their conclusion was that the structure of the wall turbulence is significantly altered by the particles resulting in a much weaker organization into coherent structures. 

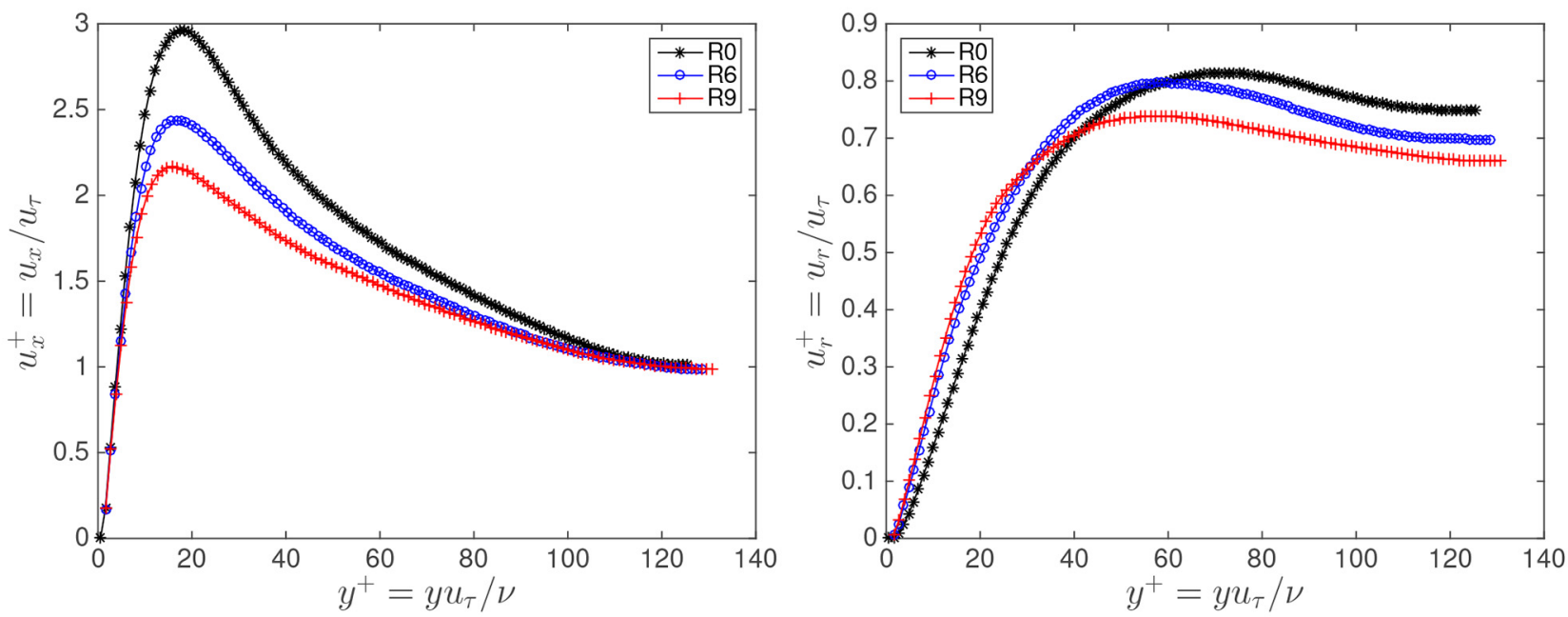

Fig. 9. RMS of the axial (left) $u_{x}^{+}$and radial (right) $u_{r}^{+}$fluctuating velocity components for the fluid phase in viscous wall units, $y^{+}$, for single-phase flow (R0) and for the case of the two particle radii (R6 and R9).

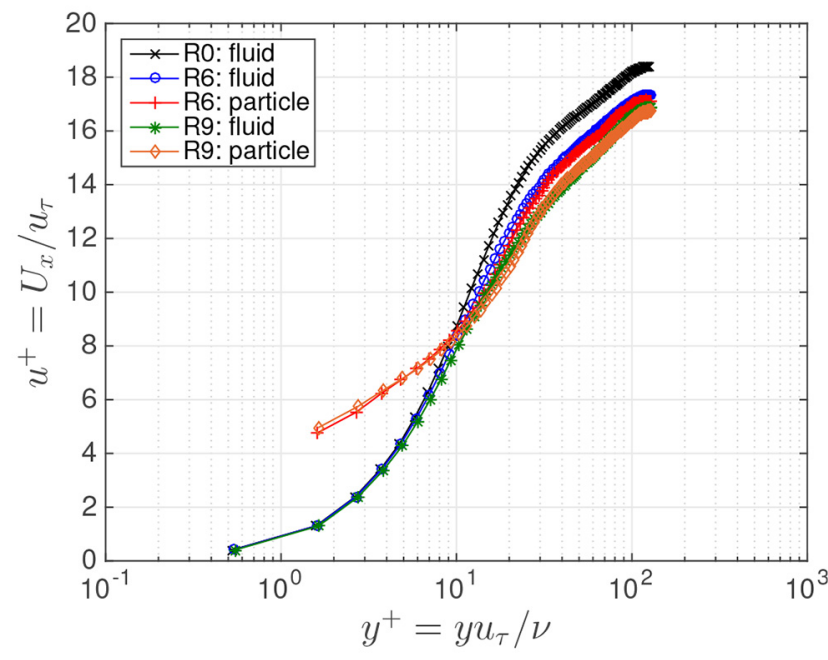

Fig. 10. Mean particle and fluid axial velocity profile $\left(U_{x}\right)$ in viscous wall units. The velocity statistics have been calculated using phase-ensemble average and rigid-body motion for lattice nodes inside the particle.

To explore this further, we look into the dissipation rate in the turbulent pipe flow. The dissipation rate, $\epsilon$, may be written down in terms of the fluctuating rates of strain obtained from the turbulent flow field and kinematic viscosity, $\nu$. By definition, the energy dissipated per unit volume is given by

$$
\epsilon=2 \nu E_{i j} E_{i j},
$$

where $E_{i j}$ is the symmetric part of the velocity gradient tensor, i.e., $E_{i j}=1 / 2\left(\partial u_{i} / \partial x_{j}+\partial u_{j} / \partial x_{i}\right)$. Using Einstein notation, and symmetry arguments for Cartesian coordinates, this expression for $\epsilon$ becomes

$$
\epsilon=2 \nu\left(E_{11}^{2}+E_{22}^{2}+E_{33}^{2}+2\left(E_{12}^{2}+E_{13}^{2}+E_{23}^{2}\right)\right) .
$$

Thus, dissipation is particularly pronounced in regions where the instantaneous gradient in velocity, and hence

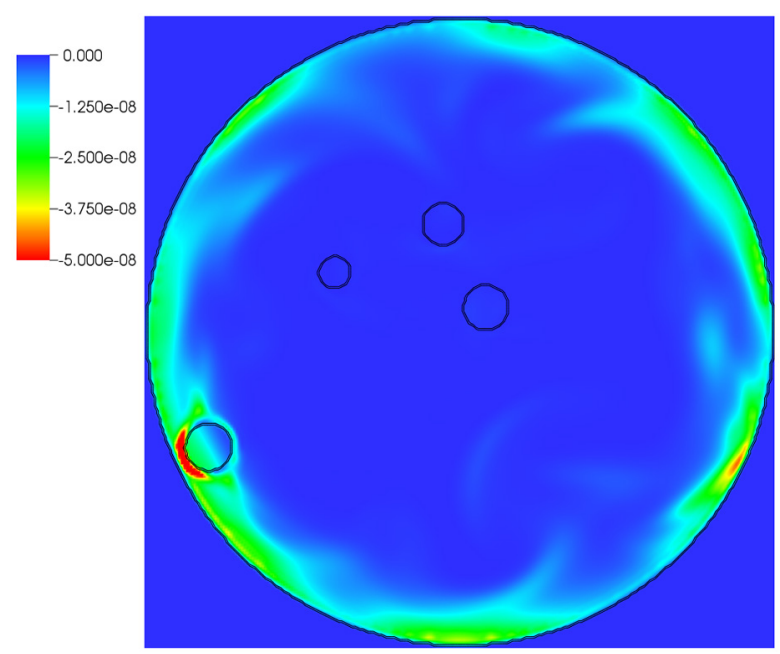

Fig. 11. Plot of energy dissipation, $\epsilon$, along the length of the pipe for the case R9 at a cross-section. The particle and pipe boundaries are marked by a black line. The fluid confined between the particle and the pipe wall shows energy dissipation as high as five times the mean value.

shear stress, is large. We now investigate the influence of particles near to the wall on the distribution of energy dissipation. In fig. 11, we plot the instantaneous energy dissipation for the case $\mathrm{R} 9$, together with the particle positions close to the wall. The plot shows a peak in the energy dissipation in regions where the fluid is confined between the particle and the wall. This suggests that high particle-to-fluid apparent slip velocity, close to the wall, corresponds locally to events of high dissipation rate. Essentially, the particle tries to displace the fluid along with it while the wall tries to bring it to a halt, resulting in large velocity gradients and hence large energy dissipation. Similar observations were made in [54] where particles have a significant slip velocity that is reflected in extreme events in the distribution of wall shear stresses. 


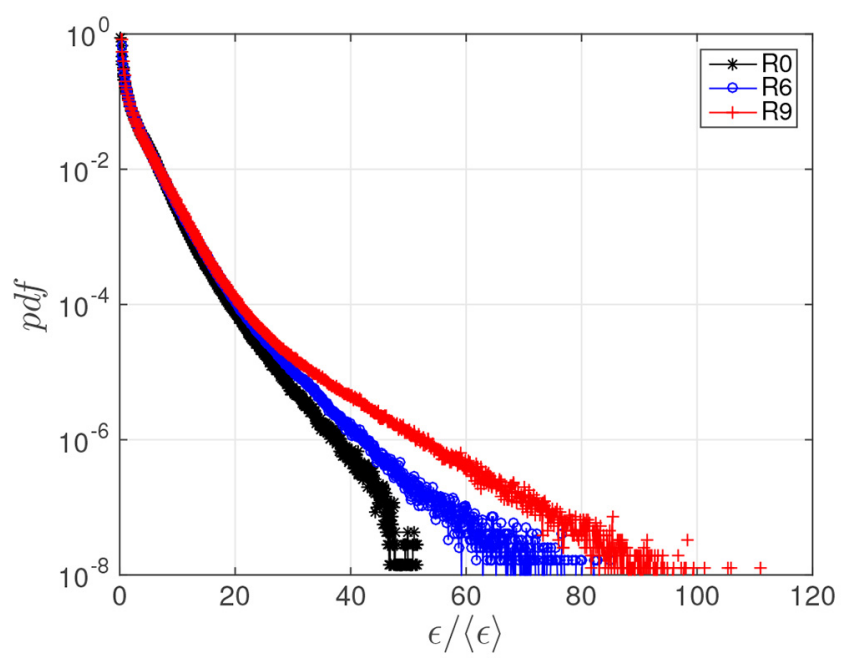

Fig. 12. Probability density function of the energy dissipation, $\epsilon$, normalized with respect to their mean, $\langle\epsilon\rangle$, for single-phase flow (R0) and for the case of the two particle radii (R6 and R9).

The probability density function (pdf) of the energy dissipation, presented in fig. 12 , shows a clear difference between the typical distribution of energy dissipation for a single-phase flow, and for the particle-laden cases. In the presence of particles, the tails of the pdf are greatly stretched, with substantial probability of finding values of the energy dissipation rate up to several times the value observed for single-phase flow. The results for small and large particles show a similar behavior with more extreme events of energy dissipation for larger particles.

\section{Structure of the dispersed phase}

Visualizations of the typical particle trajectories are reported in fig. 13. The irregular motion of the particles spanning the entire cross-section of the pipe can be clearly observed.

Figure 14 and 15 show the results for the joint probability distribution function (pdf) of axial and angular velocity, respectively, with radial position of the center of mass of the particles obtained from the simulation. The axial velocity is normalized by the friction velocity of the corresponding simulation, the angular velocity is normalized by the ratio of friction velocity to the pipe radius and the radial positions are normalized with the radius of the pipe.

The particle axial velocity is compared with the mean Eulerian velocity obtained from another simulation without particles but with the same energy input. It is observed that the axial velocity of particles is scattered around the mean velocity obtained from the Eulerian field. The angular velocity of a sphere in a shear flow with shear rate $G$, is given by $G / 2$. For this reason, the magnitude of angular velocities of the particles is compared with the half of the shear rate $(G / 2)$, again obtained from another simulation with the same energy input but without particles. The angular velocities of the particles increase towards the wall due to the presence of a high-shear region and remain scattered around the values of shear rate obtained from the Eulerian field. The finite size of the particles results in an excluded region near the wall which increases for higher particle radii.

\subsection{Stresslet and fragmentation rate}

In order to quantify the effect of a straining flow field on particles, we compute the stresslet (the symmetric component of the first moment of the force per unit area) on the surface of the particles:

$$
S_{i j}=\int_{S_{p}}\left[\sigma_{i k} x_{j}+\sigma_{j k} x_{i}\right] n_{k} \mathrm{~d} s-\frac{1}{3} \int_{S_{p}} \sigma_{i k} x_{j} \delta_{j k} n_{k} \mathrm{~d} s,
$$

where $x_{j}$ is the vector connecting the surface element to the center of mass of the particle, $n_{k}$ is the outward unit normal from the particle surface, $\sigma_{i k}$ is the fluid stress tensor and the integration takes place over the surface of the particle $S_{p}$. The computation of forces locally on the surface of the particle using the bounce-back scheme enables us to compute the stresslet directly.

A definition of the fragmentation rate in terms of first exit-time statistics was proposed in [32]. This corresponds to the measurement of fragmentation rate by computing the average time that it takes for the particles to reach the first occurence of local hydrodynamic stresses that are strong enough to break the particle. They define fragmentation as an instantaneous event in time. Here we assume that this happens when the local stresslet, acting on the particle, exceeds a critical $S T_{c r}$, i.e., we consider the limit of highly brittle particles, which is believed to hold for small and compact particles made of materials that form stiff bonds. In this limit, the time for accumulating the stress is small compared with the time over which the stress is applied so that with respect to the time scale of the stress fluctuations, fragmentation occurs instantaneously. The critical stresslet is a characteristic of the particle under consideration, i.e., $S T_{c r}$ is a function of the particle properties such as size, structure, type of constituent particles, and chemical environment.

In fig. 16, we show schematically the way in which we propose to estimate the fragmentation rate, using a real example taken from the evolution of radial position (left) and stresslet (right) of two particles. In fig. 16 (right), we show the time series of the stresslet norm $(S T)$ along a particle trajectory and the procedure followed to define the exit-time. Assuming a particle released at a time $t_{0}$ moves with the flow for a time $t_{\text {frag }}$ after which the stresslet norm exceeds, for the first time, the critical threshold $S T_{c r}$ (indicated by the horizontal line in fig. 16) where the particle breaks up. The first crossing of $S T_{c r}$ thus defines the exit time $\tau\left(S T_{c r}\right)$, which is the basic quantity for determining the fragmentation rates. It should be noted here that the term fragmentation is slightly inappropriate from the perspective of our simulations since we are using rigid non-breaking spheres. However, we can still define the fragmentation rate as the average time after which the particle with given critical stresslet $S T_{c r}$, 

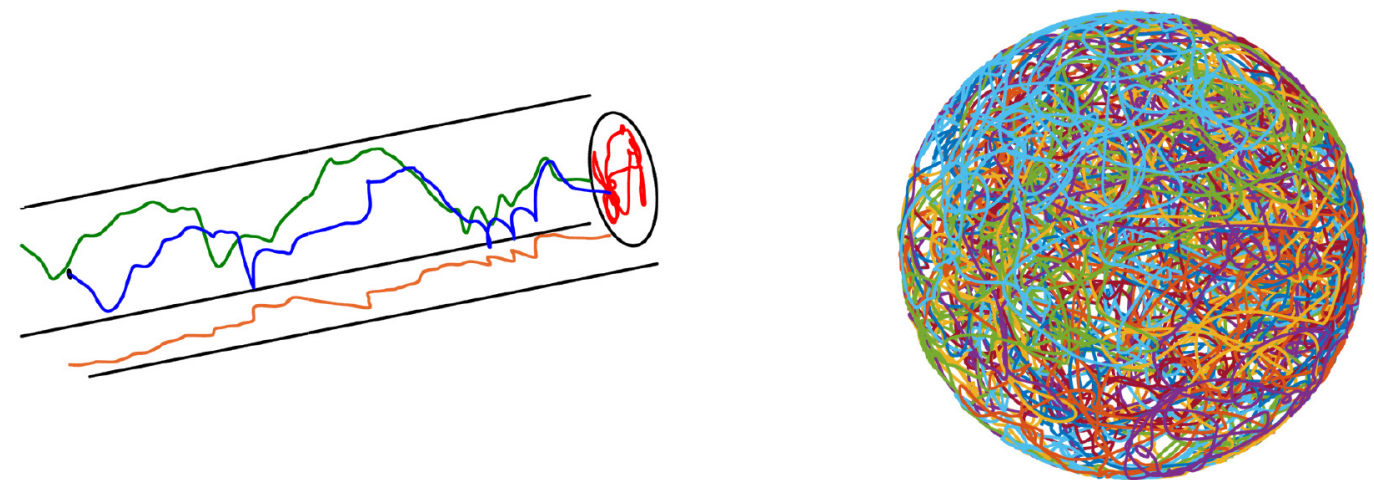

Fig. 13. Left: trajectory of a particle in the turbulent pipe for ten eddy turnover times, $t^{+}=R / u_{\tau}$, with its projection on three orthogonal planes. The black line indicates the projection of boundaries of the pipe on the orthogonal planes. For the purpose of visualization, the axial direction has been contracted by a factor of 20. Right: axial view of ten particle trajectories in the turbulent pipe for ten eddy turnover times (different colors correspond to different particle trajectories).
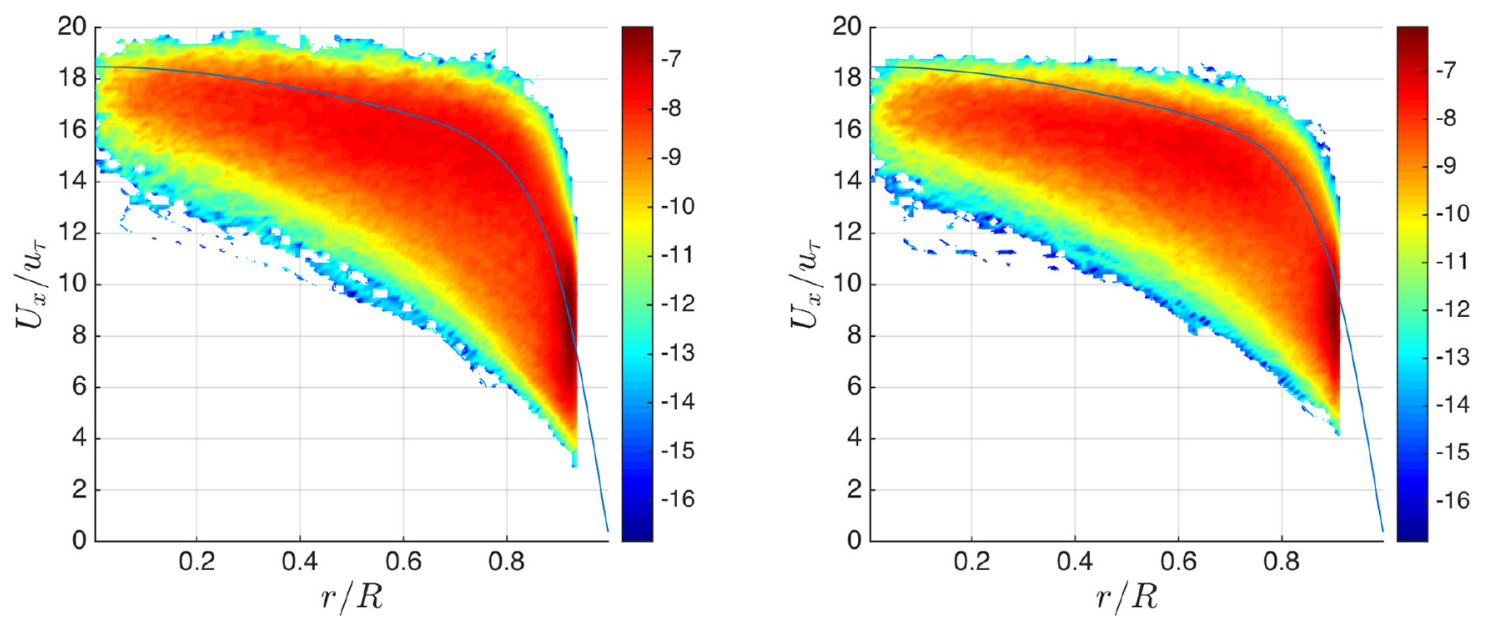

Fig. 14. Joint PDF of axial velocity for R6 (left) and R9 (right) with radial position of the particles. Color bar indicates $\log _{10}$ values of the probability. Blue lines indicate the mean axial velocity obtained from the Eulerian field of a turbulent pipe flow simulation without particles but with the same energy input as the one with particles (R0).
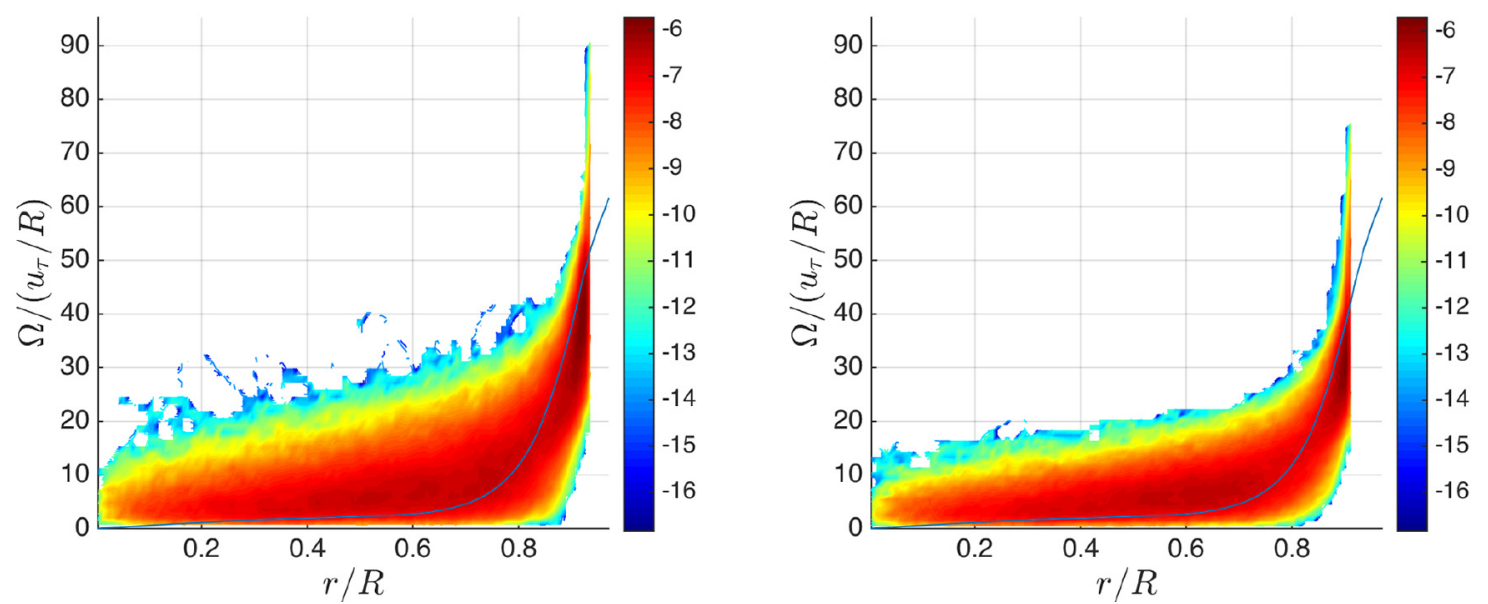

Fig. 15. Joint pdf of the magnitude of the angular velocity, for R6 (left) and R9 (right), and of the radial position of the particles. Color bar indicates $\log _{10}$ values of the probability. Blue lines indicate half of the mean shear rate obtained from the Eulerian field of the turbulent pipe flow simulation without particles but with the same energy input as the one with particles (R0). 

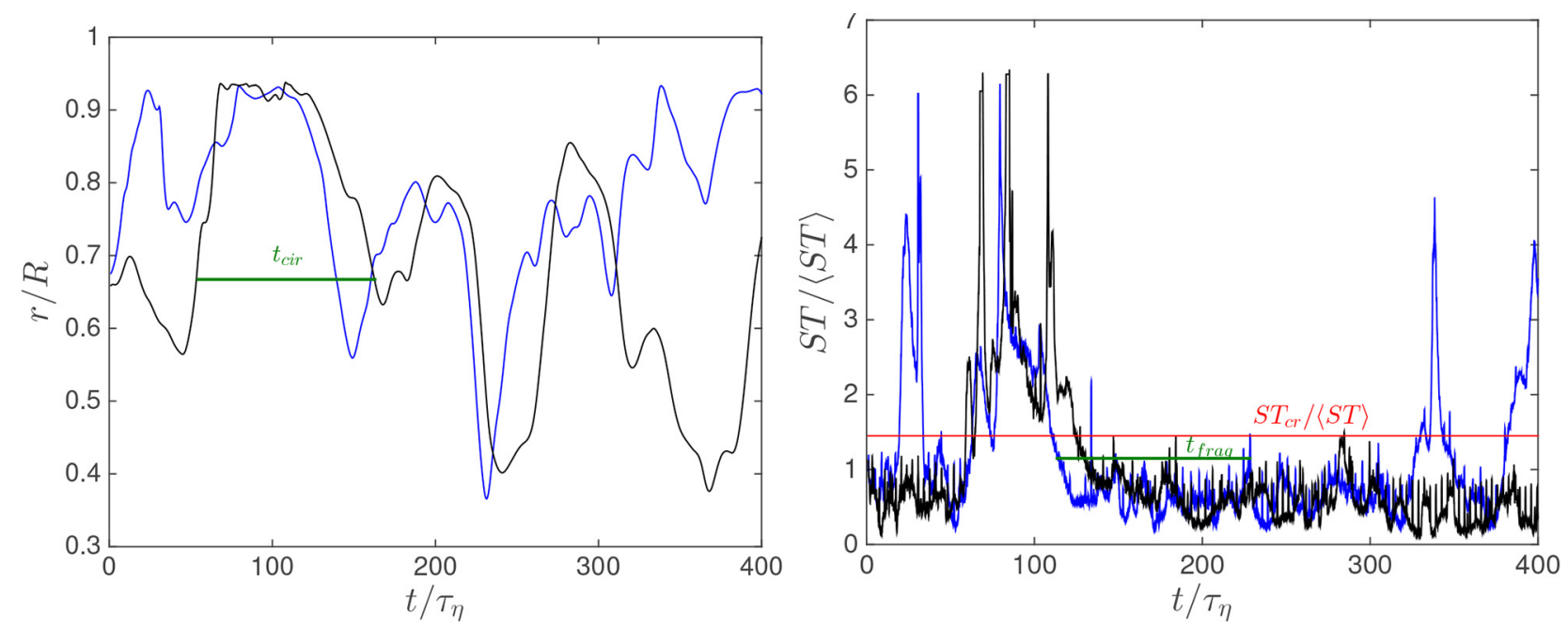

Fig. 16. Time evolution of the particle radial position (left) and of the corresponding stresslet norm (right) for two sample particles. Time $t$ is normalized by the Kolmogorov time, radial positions are normalized by the pipe radius and the stresslet norm is normalized by its global mean.

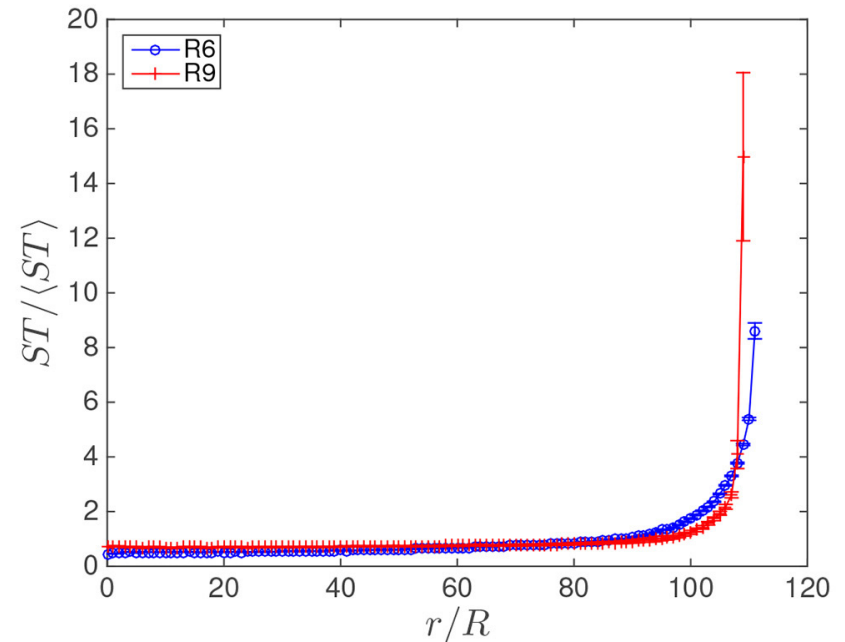

Fig. 17. Mean stresslet norm $(S T)$ normalized with its global mean, $\langle S T\rangle$, as a function of the radial distance in the turbulent pipe flow for the two particle radii (R6 and R9) with error bars representing the RMS of stresslet fluctuations.

would have been damaged. The fact that our particles remain intact, and continue to move, allows us to collect more statistics by randomly choosing the release time, $t_{0}$, multiple times per particle and then further averaging the data over multiple particles.

The fragmentation rate $\left(f_{\text {frag }}\right)$ for the given threshold of stresslet $\left(S T_{c r}\right)$ is then given by the inverse of the mean exit-time, computed as the ensemble average over many trajectories:

$$
f_{\text {frag }}=\frac{1}{\left\langle\tau\left(S T_{c r}\right)\right\rangle_{\text {exit }}} .
$$

From fig. 16, where we plot the time evolution of the particle radial position (left) and of the corresponding stresslet norm, it appears that particles are subject to high fluctuations of the stresslet even when in the bulk of the

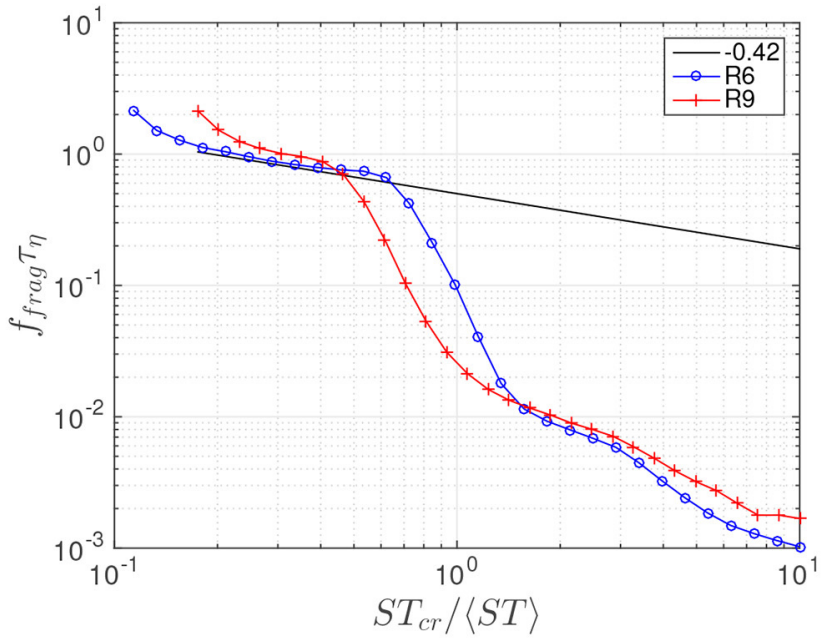

Fig. 18. Fragmentation rate, $f_{\text {frag }}$, as a function of the critical stresslet values, $S T_{c r}$, for R6 and R9 non-dimensionalized using the Kolmogorov time scale, $\tau_{\eta}$, and global mean of stresslet norm, $\langle S T\rangle$, respectively, along with the scaling exponent 0.42 corresponding to a fit of the right tail of the quasi-Eulerian proxy for aggregate break-up [32], which well describes fragmentation in HIT.

pipe while the stresslet assumes its highest values close to the wall. The discussion can be quantified by looking at the time-averaged profiles of the stresslet norm in the turbulent pipe flow measured as shown in fig. 17. Close to the wall, the stresslet assumes large values, which are an order of magnitude higher than the corresponding mean, due to high shear and wall effects. The stresslet does not decay to zero near the center of the pipe, probably due to turbulent velocity fluctuations and exhibits a flatter profile.

Figure 18 show the fragmentation rate for the two particle radii along with a scaling exponent of 0.42 . The latter corresponds to a numerical fit of $0.42 \pm 0.02$ observed for the fragmentation rate in homogeneous isotropic turbu- 

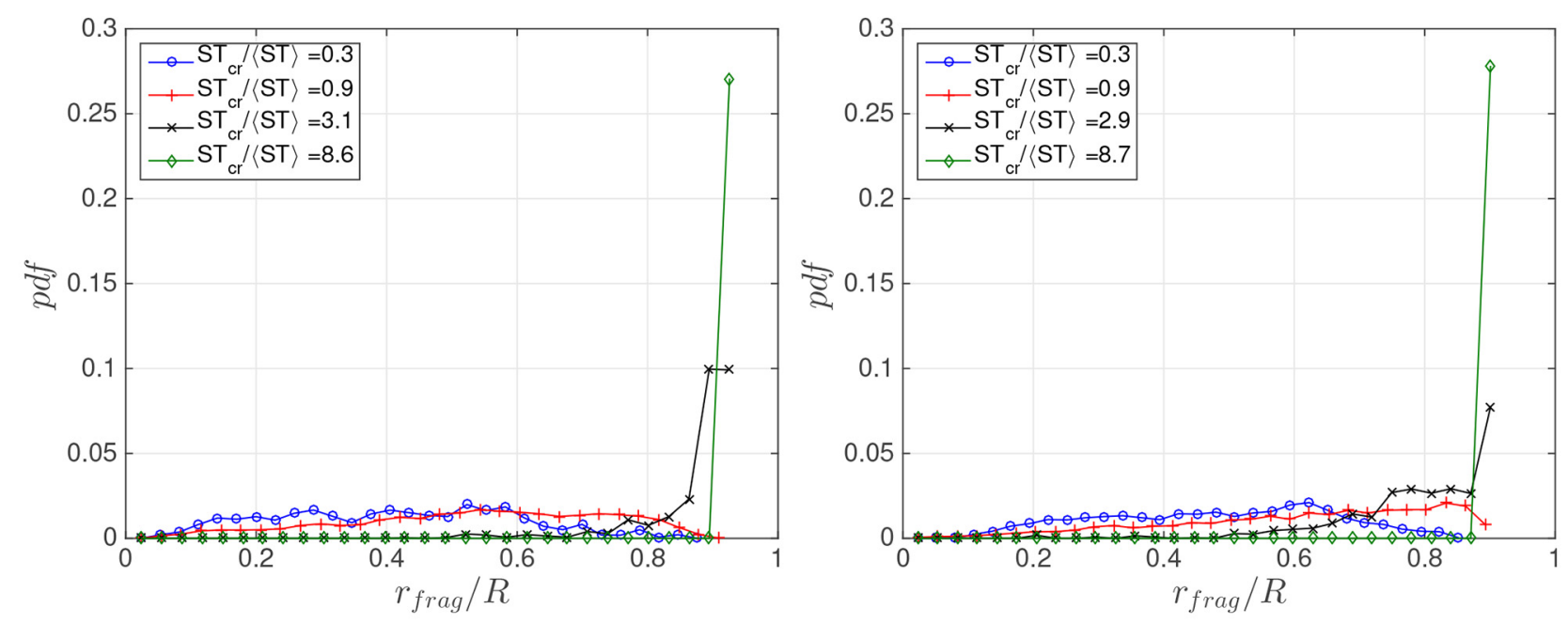

Fig. 19. Probability distribution of the radial position where fragmentation occurs, $r_{\text {frag }}$, normalized by the radius of the pipe, $R$, in the turbulent pipe flow for R6 (left) and R9 (right) for different values of the critical stresslet, $S T_{c r}$.

lence [33]. As expected, the fragmentation rate decreases with increasing values of critical stresslet $\left(S T_{c r}\right)$, confirming earlier results suggesting that weak particles break faster than small ones, as the particles with lower values of $S T_{c r} /\langle S T\rangle$ (weak particles) have a higher value $f_{\text {frag }} \tau_{\eta}$ (faster breakage). Furthermore, for small $S T_{c r}$, the fragmentation rate shows a power-law-like behavior that is similar amongst different datasets. Power-law fragmentation rates have been proposed for describing the evolution of the particle size distribution in the framework of population balance models, where they lead to reasonable agreement with experiments, at least within a certain range of experimental parameters [55]. The observed power-law scaling corresponds to the fast break-up of particles as they start from a region of where the stresslet is close to critical values. Deviations from the plateau are seen for larger values of $S T_{c r}$. For these values of $S T_{c r}$, the particles in the bulk first get entrained by turbulent eddies that transport them to the wall. During this entrainment particles gradually experience larger values of stresslet (fig. 16).

For larger threshold values, again a leveling-off in the decrease of the fragmentation rate is observed. This is in contrast to the homogenous flows, for which fragmentation shows a strong drop-off at large critical values [33]. The higher fragmentation rates for wall-bounded flows are due to the high stresslet close to the wall, which causes particles coming close to the wall to rapidly break up. In the homogenous flows, strong aggregates are only broken by the rare excursions of dissipation from the mean caused by intermittency $[32,33]$. As these events are rare, the fragmentation rate exhibits a superexponential dropoff for large dissipation. The time difference between the first plateau and the leveling-off corresponds to the timescale of the movement of particles from the bulk to the near-wall regions.

The observation made from fig. 18 suggests that weak particles (small $S T_{c r}$ ) in the wall-bounded flows are bro- ken by turbulent fluctuations shortly after their release, while on the other hand strong particles (large $S T_{c r}$ ) survive for a longer time, during which they move further downstream where they eventually gets fragmented. To explore this further, we examined the spatial location at which fragmentation occurs in the wall-bounded flows. Figure 19 shows the pdf of average radial coordinates at which fragmentation occurs for different critical stresslet values. As can be seen, with increasing critical values of stresslet, the particles on average move closer to the wall before fragmenting. The average fragmentation location for weak particles is therefore close to the average location of where the particles were released. It can be seen that weak particles (small $S T_{c r}$ ) are homogeneously fragmented, whereas strong particles (large $S T_{c r}$ ) move radially and predominantly break close to the wall. The fact that the fragmentation rate computed from our simulations shows scaling corresponding to the HIT case for small critical values of stresslet, despite the finite-size particles and the different method to quantify to fragmentation, suggests that the microscopic mechanism causing fragmentation is consistent over a wide range of size and stress magnitude.

\subsection{Particle migration}

In this section, we discuss the migration of particles in the turbulent pipe flow. As discussed in sect. 1, particles migrate in turbulence due to the influence of eddies of various sizes, instead of accumulating at an equilibrium position as in a laminar flow. In the photo-bioreactor literature $[6,56]$, researchers approximate the friction velocity of the turbulent pipe flow as the radial velocity.

$$
U_{R}=u_{\tau}=\sqrt{\frac{\tau_{w}}{\rho}},
$$

where $\tau_{w}$ is the wall shear stress and $\rho$ is the fluid density. 
We compute the particle migration statistics inspired by the technique used in the previous section to measure the exit-time statistics. Here, instead of the stresslet, we use the radial position of particles shown in fig. 16. To measure the migration statistics of the particles, we compute the average time that the particle takes to return to the same radial position (return-time statistics), starting randomly from any radial position. The following protocol is applied: i) for each particle trajectory, select a starting time $t_{0}$ at random. ii) Note that the radial position $\left(r_{0}\right)$ of a particle at $t_{0}$ defines a bin of size $\Delta_{r}$ lattice units around the radial position. iii) The particle is then followed over time until it reaches again the same radial position $\left(r_{0}-\Delta_{r} \leq r_{p} \leq r_{0}+\Delta_{r}\right)$, e.g., $t_{c i r}$ in fig. 16 . The circulation frequency, $f_{c i r}$, for the given radial position is then given by the inverse of the mean of the return time, computed as the ensemble average over many time histories using a single particle trajectory and then averaged over all particles. Figure 20 shows the circulation frequency computed in this way for R6 and R9 compared with the frequency estimated using the friction velocity and pipe radius as velocity and length scales, respectively. The results are normalized with the Kolmogorov time and the pipe radius. It can be observed that using the actual radial velocity fluctuation can improve the prediction of circulation frequency, as apparent from the correlation between fig. 9 and fig. 20. It can be further observed that a maximum exists near the wall which probably corresponds to the maximum in the radial velocities of the Eulerian field. The radial position of the maxima seems to shift away from the wall for larger particle radius. However, simulations at several different particle radii with larger statistics are needed to accurately quantify the effect of particle size on radial migration. The measurements also suggest that the friction velocity slightly over-predicts the radial velocity in the turbulent pipe.

To further understand the dynamics of the interactions in the flow, we studied the radial particle dispersion. The hydrodynamic, particle-particle and particle-wall interactions induce lateral forces and diffuse the particles away from their initial path. Here, we compute the meansquare radial displacement of the particle trajectories as a function of the time interval, averaging over all times and particles, sampling after the initial transient in the flow development. The radial mean-square particle displacement, defined by eq. (14),

$$
M S D(\Delta t)=\left\langle\Delta r^{2}\right\rangle=\left\langle[r(t+\Delta t)-r(t)]^{2}\right\rangle_{p, t},
$$

where the subscript, $p, t$ means that the averaging is done for all the particles and for the complete length of the trajectory of each particle (excluding the transient period). The computed radial mean-square particle displacement normalized with the pipe radius squared is displayed as a function of $\Delta t$ in fig. 21 for R6 and R9.

For both the cases studied, the mean-square dispersion grows initially quadratically for both particles. In this ballistic regime, the particle trajectories are correlated and the displacements are proportional to $\Delta t$. The trend changes for larger time lags when the dispersion becomes negligible as the curve flattens. This is induced by

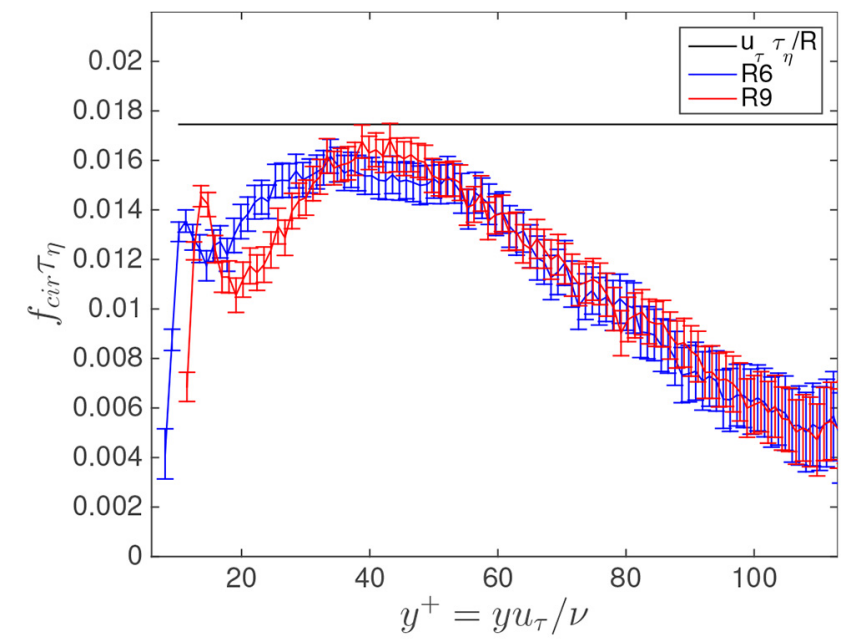

Fig. 20. Particle circulation frequency, $f_{\text {cir }}$, nondimensionalized using Kolmogorov time scale, $\tau_{\eta}$, in a turbulent pipe flow for R6 and R9 along with the frequency computed using friction velocity, $u_{\tau}$, and pipe radius. The size of the error bars is equal to the corresponding values in the bin divided by the square root of the number of entries in that bin.

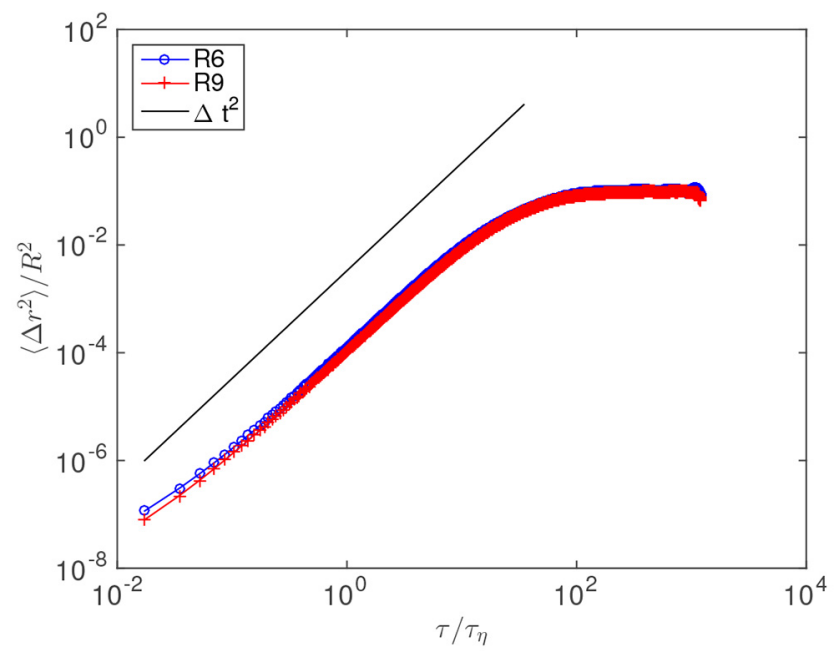

Fig. 21. Mean-square particle displacement in the radial direction non-dimensionalized using the square of the radius of the pipe in a turbulent pipe flow for R6 and R9 as a function of time, non-dimensionalized using the Kolmogorov time scale.

particle-particle and more importantly particle-wall collisions that de-correlate the trajectories in time. At higher density, it should take longer to diffuse a given distance, as other particles continually impede its progress. However, the two cases considered shows similar radial dispersion characteristics suggesting that the higher volume fraction for R9 does not significantly affect its radial dispersion. In addition to radial migration, it is also important to study the tangential migration in a photo-bioreactor. This is because the availability of light in a photo-bioreactor has a strong angular dependence due to the orientation of the Sun with respect to the reactor, which further depends on the time of the day, the geographic location of the reactor, 


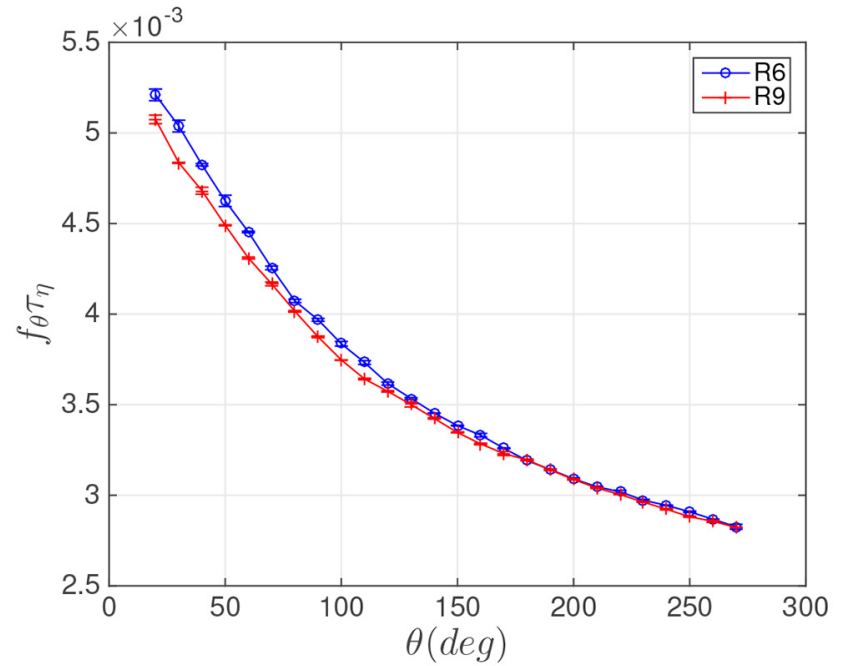

Fig. 22. Particle tangential circulation frequency, $f_{\theta}$, nondimensionalized using the Kolmogorov time scale, $\tau_{\eta}$, in a turbulent pipe flow for R6 and R9.

the surface slope, and the inclination of photo-bioreactors with respect to the surface. Consequently, the algae experiences strong gradients of light intensity as it travels tangentially in the reactors. We approximate the dark zone as sector subtending an angle $\theta$ at the center of the pipe. We computed the particle tangential circulation frequency as a function of angle $\theta$, in a similar way as we computed the radial circulation frequency. We computed the tangential circulation frequency using the average time that it took a particle starting from the center of dark zone to go the light zone and then come back to the dark zone as a function of the angle $\theta$ and the results are shown in fig. 22. It can be observed that the tangential circulation frequency for R6 is slightly higher for smaller $\theta$ while both cases exhibit the same frequency for larger values of $\theta$. From an application perspective, the knowledge of these statistics is extremely relevant for optimizing the light-dark cycles in photo-bioreactors.

\section{Conclusions}

The present work outlines a novel approach of using stresslet, computed from a finite-size representation of particles, in combination with a Direct Numerical Simulation (DNS) of turbulence to study the phenomenology of particles fragmentation in turbulent flow. The outcome of our analysis is a fully Lagrangian definition of the fragmentation rate, depending on the critical values of the stresslet, which models the strength of the particles (against fragmentation). From the perspective of the application to photo-bioreactors, algae strength can be measured in terms of the critical stresslet values (discussed in sect. 4.1) by correlating experiments in simple shear devices [5] with numerical simulations under similar flow conditions. The fragmentation rate was observed to exhibit scaling corresponding to homogenous isotropic turbulence, for small critical values, and a transition region, for larger critical values of stresslet, which corresponds to the transfer of particles from the center of the pipe to the near-wall regions. Besides computing the stresslet and the fragmentation rate of the particles, we also looked at the dynamics of particles and at the statistics of the Eulerian velocity field. It was observed that unlike the fluid, particle velocity does not vanish in the near-wall region. Large particle-to-fluid slip velocity was observed close to the wall, which corresponds locally to events of high energy dissipation.

We use the straining flow quantified using the stresslet, to compute the statistics of cell damage. In addition to the straining flow as a source of deformation, pressure difference along the cell surface might be also involved in cell damage. However, the pressure difference across the cell wall depends on the internal structure and metabolism of the cell and can vary from species to species. Alternatively, one can compute the pressure difference across the diametrically opposite points on the surface of the cell and then compute the integral of the absolute value of this quantity over the surface. Statistics of pressure difference to understand cell damage can be used to complement the analysis presented here and will be discussed in further research.

The shortcoming of the method is that we used rigid particles for representing algae and assumed a brittle fragmentation. The methodology can be further improved by the use of deformable particles using combination of LBM with immersed boundary [57] or finite-element methods [58] and using appropriate assumptions for the fragmentation process, based on the individual algae characteristics. Another shortcoming stems from the multiscale nature of the problem. The ratio of algae to photobioreactor diameter is about $10^{-4}$ [8]. This requires a resolution of $10^{5}$ grid points in lateral directions (assuming the particle diameter is represented by 10 grid points), which is computationally out of reach. However, the knowledge of the multi-scale statistics of turbulent fluctuations obtained from such simulations, down to the individual alga, is key to develop models necessary to up-scale photobioreactors, select algae strain, optimize algae productivity and reduce bioreactors energy consumption. Overall, the method provides a versatile, and numerically efficient tool, for the study of suspension of particles in turbulent flows.

This work is part of the Industrial Partnership Programme (IPP) Computational Sciences for Energy Research of the (former) Foundation for Fundamental Research on Matter (FOM), which is (now) part of the Netherlands Organization for Scientific Research (NWO). This research programme is cofinanced by Shell Global Solutions International B.V. The authors gratefully acknowledge the support of the NWO for the use of supercomputer facilities (Cartesius) under Grant No. SH-334-15. The authors would also like to thank Matthäus U. Bäbler from KTH Royal Institute of Technology and Wim-Paul Breugem from TU Delft for the interesting scientific discussions. Finally, the authors would like to thank their colleagues Gianluca Di Staso and Pinaki Kumar from TU Eindhoven for the numerous scientific discussions that greatly helped to improve the quality of the manuscript and to Xiao Xue for improving the particle update algorithm. 


\section{Author contribution statement}

FT and $\mathrm{HC}$ devised the project and conceived the original idea of the study. AG developed the necessary code, performed the numerical simulations and data analysis. All authors discussed the results and contributed to the final manuscript. AG drafted the first version of the manuscript with support from FT and HC. All the authors have read and approved the final manuscript.

Open Access This is an open access article distributed under the terms of the Creative Commons Attribution License (http://creativecommons.org/licenses/by/4.0), which permits unrestricted use, distribution, and reproduction in any medium, provided the original work is properly cited.

\section{References}

1. C.T. Crowe, J.D. Schwarzkopf, M. Sommerfeld, Y. Tsuji, Multiphase Flows with Droplets and Particles (Taylor \& Francis, 1997).

2. C.E. Brennen, Fundamentals of Multiphase Flow (Cambridge University Press, 2005).

3. O.N. Ross, Algal Motility in Variable Turbulence, PhD Thesis, University of Southampton Abstract Faculty, 2004.

4. C. Gudin, D. Chaumont, Bioresour. Technol. 38, 145 (1991).

5. M.H.A. Michels, A.J. van der Goot, N.H. Norsker, R.H. Wijffels, Bioprocess Biosyst. Eng. 33, 921 (2010).

6. E. Molina Grima, F.G. Acién Fernández, F. García Camacho, Yusuf Chisti, J. Biotechnol. 70, 231 (1999).

7. A. Contreras, F. Garca, E. Molina, J.C. Merchuk, Biotechnol. Bioeng. 60, 317 (1998).

8. E. Molina, J.M. Fernandez-Sevilla, G. Acien, Microalgae, mass culture methods, Encyclopedia of Industrial Biotechnology (John Wiley \& Sons, 2010) pp. 1-24.

9. L.I. Zaichik, V.M. Alipchenkov, E.G. Sinaiski, Particles in Turbulent Flows (Wiley, 2008).

10. F. Toschi, E. Bodenschatz, Annu. Rev. Fluid Mech. 41, 375 (2009).

11. S. Balachandar, John K. Eaton, Annu. Rev. Fluid Mech. 42, 111 (2010).

12. A. Gupta, H.J.H. Clercx, F. Toschi, Commun. Comput. Phys. 23, 665 (2018).

13. M. Uhlmann, Investigating turbulent particulate channel flow with interface-resolved DNS, in 6th International Conference on Multiphase Flow, ICMF 2007, Leipzig, Germany, July 200\%, edited by M. Sommerfeld, http:// www-cfd.ifh.uni-karlsruhe.de/uhlmann/particle/ report/icmf07.pdf.

14. M. Uhlmann, Phys. Fluids 20, 053305 (2008).

15. A.G. Kidanemariam, C. Chan-Braun, T. Doychev, M. Uhlmann, New J. Phys. 15, 025031 (2013).

16. X. Shao, T. Wu, Z. Yu, J. Fluid Mech. 693, 319 (2012).

17. I. Lashgari, F. Picano, W.P. Breugem, L. Brandt, Phys. Rev. Lett. 113, 254502 (2014).

18. F. Picano, W.P. Breugem, L. Brandt, J. Fluid Mech. 764, $463(2015)$.

19. W. Fornari, A. Formenti, F. Picano, L. Brandt, Phys. Fluids 28, 033301 (2016).
20. P. Costa, F. Picano, L. Brandt, W.P. Breugem, Phys. Rev. Lett. 117, 134501 (2016).

21. I. Lashgari, F. Picano, L. Brandt, Theor. Appl. Mech. Lett. 5, $121(2015)$.

22. V. Loisel, M. Abbas, O. Masbernat, E. Climent, Phys. Fluids 25, 123304 (2013)

23. Z. Yu, T. Wu, X. Shao, J. Lin, Phys. Fluids 25, 43305 (2013).

24. F. Janoschek, F. Toschi, J. Harting, Phys. Rev. E 82, 056710 (2010).

25. F. Janoschek, F. Toschi, J. Harting, Macromol. Theory Simul. 20, 562 (2011).

26. T. Krüger, B. Kaoui, J. Harting, J. Fluid Mech. 751, 725 (2014).

27. M. Do-Quang, G. Amberg, G. Brethouwer, A.V. Johansson, Phys. Rev. E 89, 013006 (2014).

28. A. Ten Cate, J.J. Derksen, L.M. Portela, H.E.A. Van Den Akker, Andreas Ten Cate, Jos J. Derksen, Luis M. Portela, Harry E.a. Van Den Akker, J. Fluid Mech. 519, 233 (2004).

29. H. Gao, H. Li, L.P. Wang, Comput. Math. Appl. 65, 194 (2013).

30. Lian-Ping Wang, Cheng Peng, Zhaoli Guo, Zhaosheng Yu, J. Fluids Eng. 138, 041306 (2015).

31. M.U. Bäbler, M. Morbidelli, Jerzy Baldyga, J. Fluid Mech. 612, 261 (2008).

32. M.U. Babler, L. Biferale, L. Brandt, U. Feudel, K. Guseva, A.S. Lanotte, C. Marchioli, F. Picano, G. Sardina, A. Soldati, F. Toschi, J. Fluid Mech. 766, 104 (2015).

33. M.U. Babler, L. Biferale, A.S. Lanotte, Phys. Rev. E 85 025301 (2012).

34. C. Marchioli, A. Soldati, Phys. Rev. E 91, 053003 (2015).

35. G. Segré, A. Silberberg, J. Fluid Mech. 14, 115 (1962).

36. G. Segré, A. Silberberg, J. Fluid Mech. 14, 136 (1962).

37. D.D. Joseph, D. Ocando, J. Fluid Mech. 454, 263 (2002).

38. J.P. Matas, J.F. Morris, E. Guazzelli, J. Fluid Mech. 515, 171 (2004)

39. J.P. Matas, J.F. Morris, E. Guazzelli, J. Fluid Mech. 621, 59 (2009).

40. S. Succi, The Lattice Boltzmann Equation for Fluid Dynamics and Beyond (Numerical Mathematics and Scientific Computation), 1st edition (Oxford University Press, 2001).

41. F.J. Higuera, S. Succi, Europhys. Lett. 8, 517 (1989).

42. F.J. Higuera, S. Succi, R. Benzi, Europhys. Lett. 9, 345 (1989).

43. Z. Guo, C. Shu, Lattice Boltzmann Method and Its Applications in Engineering (World Scientific Publishing Company, 2013).

44. S.K. Kang, Y.A. Hassan, J. Comput. Phys. 232, 100 (2013).

45. A.J.C. Ladd, J. Fluid Mech. 271, 285 (1994).

46. C.K. Aidun, Y. Lu, E.J. Ding, J. Fluid Mech. 373, 287 (1998).

47. Y. Chen, Q. Cai, Z. Xia, M. Wang, S. Chen, Phys. Rev. E 88, 013303 (2013).

48. M.P. Allen, D.J. Tildesley, Computer Simulation of Liquids (Clarendon Press, New York, USA, 1989).

49. Chen-Jung Lin, James H. Peery, W.R. Schowalter, J. Fluid Mech. 44, 117 (1970).

50. H.A. Stone, J.F. Brady, P.M. Lovalenti, Inertial effects on the rheology of suspensions and on the motion of individual particles, preprint. 
51. Duane R. Mikulencak, Jeffrey F. Morris, J. Fluid Mech. 520, 215 (2004).

52. J.G.M. Eggels, F. Unger, M.H. Weiss, J. Westerweel, R.J. Adrian, R. Friedrich, F.T.M. Nieuwstadt, J. Fluid Mech. 268, 175 (1994).

53. T.-H. Wu, X.-M. Shao, Z.-S. Yu, J. Hydrodyn. 23, 21 (2011).

54. P. Costa, F. Picano, L. Brandt, W.P. Breugem, Effects of the finite particle size in turbulent wall-bounded flows of dense suspensions, arXiv:1703.06036 [physics.flu-dyn].
55. M.U. Babler, M. Morbidelli, J. Colloid Interface Sci. 316, 428 (2007).

56. E. Molina, F.G. Acién Fernández, F. García Camacho, F. Camacho Rubio, Y. Chisti, J. Appl. Phycol. 12, 355 (2000).

57. T. Kruger, F. Varnik, D. Raabe, Comput. Math. Appl. 61, 3485 (2011) (Mesoscopic Methods for Engineering and Science, Proceedings of ICMMES-09).

58. R.M. MacMeccan, J.R. Clausen, G.P. Neitzel, C.K. Aidun, J. Fluid Mech. 618, 13 (2009). 